TRANSACTIONS OF THE

AMERICAN MATHEMATICAL SOCIETY

Volume 361, Number 6, June 2009, Pages 2805-2830

S 0002-9947(09)04928-9

Article electronically published on January 26, 2009

\title{
SEMIPROJECTIVITY FOR CERTAIN PURELY INFINITE $C^{*}$-ALGEBRAS
}

\author{
JACK SPIELBERG
}

\begin{abstract}
It is proved that classifiable simple separable nuclear purely infinite $C^{*}$-algebras having finitely generated $K$-theory and torsion-free $K_{1}$ are semiprojective. This is accomplished by exhibiting these algebras as $C^{*}$ algebras of infinite directed graphs.
\end{abstract}

\section{INTRODUCTION}

The notion of semiprojectivity for $C^{*}$-algebras was introduced in [7, with the aim of extending shape theory to the noncommutative setting. An algebra was defined to be semiprojective if it exhibited homotopy continuity for maps into an inductive limit $C^{*}$-algebra. The definitions of semiprojectivity and shape theory were modified in [1]. For both definitions, the class of algebras most easily shown to be semiprojective were the Cuntz-Krieger algebras [6]. In effect, the fundamental perturbation lemmas of Glimm 9 show that the property of being a partial isometry, and the properties of equality/orthogonality for two projections, are liftable in the appropriate sense. Since Cuntz-Krieger algebras are defined by finitely many generators and relations of these types, it is easy to see that they are semiprojective.

The work of Kirchberg, Phillips and Rørdam has brought attention to a class of $C^{*}$-algebras defined by the essential features of the Cuntz-Krieger algebras, namely, the simple separable nuclear purely infinite $C^{*}$-algebras. In the presence of the universal coefficient theorem, it was shown that these algebras were classified (up to strong Morita equivalence) by $K$-theory [10, [12, and that the $K$-theory can be any pair of countable abelian groups. It is natural to ask whether these algebras also are semiprojective. Blackadar has shown that finitely generated $K$-theory is necessary for semiprojectivity 2, Corollary 2.11]. In the same paper he established semiprojectivity in the case where the $K_{0}$-group is free and the $K_{1}$-group is trivial. His arguments were generalized by Szymanski [18 to the case where the $K_{0}$-group is finitely generated and the $K_{1}$-group is free with $\operatorname{rank} K_{1} \leq \operatorname{rank} K_{0}$. In this paper, we establish semiprojectivity without the restriction on the ranks of the $K$-groups. In particular we show that the algebra $P_{\infty}$ having $K_{0}=0$ and $K_{1}=\mathbf{Z}$ is semiprojective. Although our proof also relies on realizing the algebras as $C^{*}$ algebras of infinite graphs, our techniques are substantially different from those in [2] and [18. We wish to draw attention to our Lemma 3.6, which we feel is a novel addition to the field of noncommutative general topology.

Received by the editors February 19, 2001 and, in revised form, August 26, 2005.

2000 Mathematics Subject Classification. Primary 46L80; Secondary 46L85, 22A22.

Key words and phrases. Simple purely infinite $C^{*}$-algebra, semiprojectivity, graph algebra.

(C)2009 American Mathematical Society

Reverts to public domain 28 years from publication 
For (irreducible) finite graphs, $K_{0}$ and $K_{1}$ have equal rank. To model the general situation we must use infinite graphs. In another paper [16] we proved that a graph $C^{*}$-algebra has an inductive limit decomposition over the (directed set of the) finite subgraphs. (Similar results have been obtained by different methods in [14. There is now a large body of work on the subject of graph $C^{*}$-algebras. A survey of this work, together with an extensive bibliography, can be found in Raeburn's recent CBMS lectures 13.) This provides a strategy for constructing infinite graphs whose $C^{*}$-algebra has specified $K$-theory, which we follow in section 2 of this paper. In particular, we provide an elegant graph whose $C^{*}$-algebra is the classifiable example having $K_{0}=(0)$ and $K_{1} \cong \mathbf{Z}$. (A row-finite graph with the same $C^{*}$-algebra was given by Neubüser in 11, but has not been found useful for proving semiprojectivity. It is also shown in [11] that the $K$-theory of irreducible Exel-Laca $C^{*}$-algebras (of infinite matrices; see [8]) can be any pair of countable abelian groups with free $K_{1}$. In [19] Szymanski proved the analogous result for $C^{*}$-algebras of row-finite graphs. A different version, for graphs that are not rowfinite, is given in [17] in the context of prime-order automorphisms of $K$-theory.) In section 3 we again use the inductive limit decomposition to prove semiprojectivity.

The figures in this paper were prepared with XY-pic.

We recall here the definition of semiprojectivity from [1] (slightly modified).

Definition. The $C^{*}$-algebra $A$ is called semiprojective if the following holds. If $B$ is a $C^{*}$-algebra containing a directed family $\mathcal{L}$ of ideals with closure $I$, and $B / I$ is isomorphic to $A$, then there is an ideal $J \in \mathcal{L}$ and a $*$-homomorphism from $A$ to $B / J$ lifting the quotient map.

Let $E$ be a directed graph with vertices $E^{0}$ and (oriented) edges $E^{1}$. We will follow the notation in [16], using $o$ and $t$ for the origin and terminus of an edge. However, we will omit the subscript + indicating (positively) oriented edges, as in this paper we will not make use of the negatively oriented ones. We will let the edges and vertices also denote the generating elements of the algebra $\mathcal{O}(E)$. Potential confusion will be avoidable from the context. We use the letters $e$ and $f$ for edges, and $u$ and $v$ for vertices in a graph. Thus the letters $e$ and $f$ will also denote partial isometries, while $u$ and $v$ will denote projections in the corresponding $C^{*}$-algebras. We will let $D$ denote the set of vertices of $E$ having infinite exit valence:

$$
D:=\left\{u \in E^{0} \mid o^{-1}(u) \text { is infinite }\right\} .
$$

We recall from [16, Theorem 2.21], the relations defining $\mathcal{O}(E)$. We refer to these as condition $(\mathcal{O})$.

Condition $(\mathcal{O})$.

(o1) $\left\{e \mid e \in E^{1}\right\}$ are partial isometries, and $\left\{u \mid u \in E^{0}\right\}$ are projections.

(o2) $u v=0$ if $u \neq v$, for all $u, v \in E^{0}$.

(o3) $e^{*} e=t(e)$, for all $e \in E^{1}$.

(o4) $o(e) \cdot e=e$, if $o(e) \in D$.

(o5) If $e \neq f$ and $o(e)=o(f) \in D$, then $e^{*} f=0$.

(o6) If $u \notin D$, then $u=\sum\left\{e e^{*} \mid e \in o^{-1}(u)\right\}$.

We will be primarily interested in graphs with special properties. We set out these properties here. We consider an infinite directed graph $E$ having a chain $F_{0} \subseteq F_{1} \subseteq \ldots \subseteq E$ of finite subgraphs with $\bigcup F_{n}=E$. Let $D$ be as above. We recall from [16, Theorem 2.34] that the inclusion of graphs $F_{n} \subseteq E$ determines a 
Toeplitz graph algebra $\mathcal{T} \mathcal{O}\left(F_{n}, S_{n}\right)$, where $S_{n}=\left\{u \in F_{n}^{0} \mid o^{-1}(u) \cap E^{1} \subseteq F_{n}^{1}\right\}$. We will let $\mathcal{T} \mathcal{O}\left(F_{n}\right)$ denote this algebra. It follows from [16, Theorem 2.20] that the subalgebra $\mathcal{T} \mathcal{O}\left(F_{n}\right)$ is generated by the edges and vertices of $F_{n}$ subject to the same relations $\mathcal{O}$, with the exception that the set $D$ above must be enlarged to include $F_{n}^{0} \backslash S_{n}$.

Remarks 1.1. We recall from [16, Theorem 2.35] that

$$
\mathcal{O}(E)=\overline{\bigcup_{n} \mathcal{T O}\left(F_{n}\right)}
$$

We recall from [16, Theorem 3.15] that if $E$ is an irreducible graph that is not a cycle, then $\mathcal{O}(E)$ is simple, nuclear, and purely infinite.

\section{Condition (a).}

(a1) $D \subseteq F_{0}^{0}$.

(a2) $F_{n}$ is irreducible and not a cycle, $n \geq 0$.

(a3) $o^{-1}\left(F_{n}^{0}\right) \cap t^{-1}\left(F_{n}^{0}\right) \cap E^{1} \subseteq F_{n}^{1}, n \geq 0$.

(a4) $o^{-1}\left(F_{n}^{0} \backslash D\right) \subseteq F_{n}^{1}, n \geq 0$.

(a5) $t^{-1}\left(F_{n}^{0}\right) \subseteq F_{n+1}^{0}, n \geq 0$.

We may loosely summarize condition (a) in the following way: $E \backslash D$ is locally finite and flows towards $D$, while $D$ points to all of the subgraphs.

\section{Condition (b).}

(b1) $K_{*}(\mathcal{O}(E))$ is finitely generated.

(b2) For each $u \in E^{0} \backslash D$ there exist $v \in F_{0}^{0} \backslash D$ and a graph isomorphism $\gamma$ : $o^{-1}(u) \rightarrow o^{-1}(v)$ such that $[t(e)]=[t(\gamma(e))]$ in $K_{0}(\mathcal{O}(E))$ for $e \in o^{-1}(u)$.

Condition (b2) is a technical aid in the proof of Theorem 3.1, and it is evident in the examples to which we will apply that theorem.

Remarks 1.2. (i) Since all $F_{n}$ are irreducible, $E$ is irreducible.

(ii) Since $F_{0}$ is finite, (a1) implies that $D$ is finite.

(iii) Assuming $F_{n+1} \neq F_{n}$ for all $n \geq 0$, it follows from (a3) that $F_{n}^{0} \backslash F_{n-1}^{0} \neq \emptyset$. Since $F_{n}$ is irreducible, then

$$
t^{-1}\left(F_{n}^{0} \backslash F_{n-1}^{0}\right) \cap o^{-1}\left(F_{n-1}^{0}\right) \neq \emptyset .
$$

By (a4) it follows that

$$
t^{-1}\left(F_{n}^{0} \backslash F_{n-1}^{0}\right) \cap o^{-1}(D) \neq \emptyset .
$$

In particular, $D \neq \emptyset$.

(iv) If $\left\{F_{n} \mid n=0,1,2, \ldots\right\}$ is replaced by a subsequence, properties (a1)-(a5) still hold.

(v) By passing to a subsequence, if necessary, we may assume that (a6) $t^{-1}\left(F_{n}^{0} \backslash F_{n-1}^{0}\right) \cap o^{-1}(u) \neq \emptyset, u \in D, n \geq 0$.

(vi) It follows from (b1) and Remark 1.1 that by enlarging $F_{0}$ we may assume (b3) $i_{*}: K_{1}\left(\mathcal{T O}\left(F_{0}\right)\right) \rightarrow K_{1}(\mathcal{O}(E))$ is onto.

Since $\mathcal{O}(E)$ is simple and purely infinite, it follows from [5] that by enlarging $F_{1}$ we may assume

(b4) If $v_{1}, v_{2} \in F_{0}^{0}$ satisfy $\left[v_{1}\right]=\left[v_{2}\right]$ in $K_{0}(\mathcal{O}(E))$, then $v_{1} \sim v_{2}$ in $\mathcal{T} \mathcal{O}\left(F_{1}\right)$. 
Remark 1.3. Because of (a1), the relations defining representations of $\mathcal{T} \mathcal{O}\left(F_{n}\right)$ are exactly the same as $(\mathcal{O})$ above (when applied to $F_{n}$ ). (In this case, the representation is faithful if and only if for each $u \in D, u \neq \sum\left\{e e^{*} \mid e \in o^{-1}(u)\right\}$. See [16, Theorem 2.20].)

\section{2. $K$-THEORY}

In Theorem 2.3 below we construct graphs whose algebras have a certain specified $K$-theory. To this end we must first compute the $K$-theory of the Toeplitz graph algebras $\mathcal{T} \mathcal{O}\left(F_{n}\right)$, and also study the maps in $K$-theory induced by the inclusions $\mathcal{T O}\left(F_{n}\right) \subseteq \mathcal{T} \mathcal{O}\left(F_{n+1}\right)$.

Let $F$ be a finite graph satisfying condition (I) of [4]. Let $S_{F} \subseteq F^{0}$, and let $\mathcal{T O}(F)$ denote $\mathcal{T} \mathcal{O}\left(F, S_{F}\right)$. We recall from [16, Theorem 2.33] that there is a short exact sequence

$$
0 \longrightarrow I_{F} \longrightarrow \mathcal{T} \mathcal{O}(F) \stackrel{\pi}{\longrightarrow} \mathcal{O}(F) \longrightarrow 0,
$$

where $I_{F} \cong \bigoplus_{S_{F}^{c}} \mathcal{K}$. We obtain a long exact sequence in $K$-theory:

$$
0 \longrightarrow K_{1} \mathcal{T} \mathcal{O}(F) \longrightarrow K_{1} \mathcal{O}(F) \stackrel{\partial}{\longrightarrow} \bigoplus_{S_{F}^{c}} \mathbf{Z} \longrightarrow K_{0} \mathcal{T} \mathcal{O}(F) \longrightarrow K_{0} \mathcal{O}(F) \longrightarrow 0 .
$$

From [4] we have $K_{*} \mathcal{O}(F)$ :

$$
\begin{aligned}
& K_{0} \mathcal{O}(F)=\mathbf{Z}^{F^{0}} /\left\{[u]=\sum_{e \in o^{-1}(u)}[t(e)]\right\}, \\
& K_{1} \mathcal{O}(F)=\left\{x \in \mathbf{Z}^{F^{0}} \mid x(u)=\sum_{e \in t^{-1}(u)} x(o(e))\right\} .
\end{aligned}
$$

For $x \in K_{1} \mathcal{O}(F)$ we will further define $x$ on $F^{1}$ by setting

$$
x(e):=x(o(e)) \text {. }
$$

Lemma 2.1. If $x \in K_{1} \mathcal{O}(F)$, then $(\partial x)_{u}=-\sum\left\{x(e) \mid e \in t^{-1}(u)\right\}$, for $u \in S_{F}^{c}$.

Proof. We will let $e \in F^{1}$ represent the corresponding generator of $\mathcal{T} \mathcal{O}(F)$, and $\widetilde{e}$ represent the generator $\pi(e) \in \mathcal{O}(F)$. For $u \in F^{0}$ let $\varepsilon_{u} \in \mathcal{T} \mathcal{O}(F)$ be given by

$$
\varepsilon_{u}=u-\sum_{e \in o^{-1}(u)} e e^{*} .
$$

Then $\varepsilon_{u} \neq 0$ if and only if $u \notin S_{F}$, and $\pi_{*}\left(\varepsilon_{u}\right)=0$ in $\mathcal{O}(F)$ for all $u$. Moreover, the classes $\left\{\left[\varepsilon_{u}\right] \mid u \in S_{F}^{c}\right\}$ form a basis for $K_{0}\left(I_{F}\right)$.

Now let $x \in K_{1} \mathcal{O}(F)$. We construct a unitary representing $x$ as in 15, Section 2]. With $|x|=\sum_{e \in F^{1}}|x(e)|$, we let $V(x) \in M_{|x|}(\mathcal{O}(F))$ be a diagonal matrix with $|x(e)|$ entries equal to $\widetilde{e}$ if $x(e)>0$, and with $|x(e)|$ entries equal to $\widetilde{e}^{*}$ if $x(e)<0$. Then $V(x)^{*} V(x)$ and $V(x) V(x)^{*} \in M_{|x|}\left(\operatorname{span}\left\{\widetilde{e e^{*}} \mid e \in F^{1}\right\}\right)$, and $1-V(x)^{*} V(x)$ is equivalent to $1-V(x) V(x)^{*}$. Let $W(x) \in M_{|x|}\left(\operatorname{span}\left\{\widetilde{e} \widetilde{e}^{*} \mid e \in F^{1}\right\}\right)$ with $W(x)^{*} W(x)=1-V(x)^{*} V(x)$ and $W(x) W(x)^{*}=1-V(x) V(x)^{*}$. Then $U(x)=$ $V(x)+W(x) \in M_{|x|}(\mathcal{O}(F))$ is unitary, and $[U(x)]=x$.

Next note that if we replace $\widetilde{e}$ by $e$ throughout, we obtain partial isometries $U_{0}(x), V_{0}(x), W_{0}(x) \in M_{|x|}\left(\mathcal{T O}(F)\right.$ ) that are lifts (modulo $M_{|x|}\left(I_{F}\right)$ ) of $U(x)$, $V(x), W(x)$. Therefore,

$$
\partial x=\left[I-U_{0}(x)^{*} U_{0}(x)\right]-\left[I-U_{0}(x) U_{0}(x)^{*}\right] .
$$


The fact that $\pi_{*} \circ \partial(x)=0$ accounts for all terms in the above involving the $e e^{*}$. What remains are the occurrences of the $\varepsilon_{u}$. We obtain

$$
\begin{aligned}
\partial x & =\left(\sum_{x(e)>0}|x(e)| \sum_{u \neq t(e)}\left[\varepsilon_{u}\right]+\sum_{x(e)<0}|x(e)| \sum_{u}\left[\varepsilon_{u}\right]\right) \\
& -\left(\sum_{x(e)>0}|x(e)| \sum_{u}\left[\varepsilon_{u}\right]+\sum_{x(e)<0}|x(e)| \sum_{u \neq t(e)}\left[\varepsilon_{u}\right]\right) \\
& =\sum_{e} x(e)\left(\sum_{u \neq t(e)}\left[\varepsilon_{u}\right]-\sum_{u}\left[\varepsilon_{u}\right]\right) \\
& =-\sum_{e} x(e)\left[\varepsilon_{t(e)}\right] \\
& =-\sum_{u \in S_{F}^{c}}\left(\sum_{e \in t^{-1}(u)} x(e)\right)\left[\varepsilon_{u}\right] .
\end{aligned}
$$

Thus we find that

$$
\begin{aligned}
K_{1}(\mathcal{T} \mathcal{O}(F)) \cong \operatorname{ker}(\partial) & =\left\{x \in \mathbf{Z}^{F^{0}} \mid \sum_{e \in t^{-1}(u)} x(e)=0, u \in S_{F}^{c}\right\}, \\
\operatorname{Im}(\partial) & =\left\{\left(\sum_{e \in t^{-1}(u)} x(e)\right)_{u \in S_{F}^{c}} \mid x \in K_{1}(\mathcal{O}(F))\right\} .
\end{aligned}
$$

Now let $F \subseteq G$ be an inclusion of finite directed graphs satisfying condition (I). We assume that $S_{F} \subseteq S_{G}$ (as would be the case if $G \subseteq E$, and $S_{F}, S_{G}$ are defined relative to $E)$. Let $i: \mathcal{T O}(F) \subseteq \mathcal{T O}(G)$ denote the inclusion.

Lemma 2.2. Let $x \in K_{1}(\mathcal{T O}(F))$. Then

$$
\left(i_{*} x\right)_{u}= \begin{cases}x(u) & \text { if } u \in F^{0}, \\ 0 & \text { if } u \in G^{0} \backslash F^{0} .\end{cases}
$$

Proof. When viewed as an element of $K_{1}(\mathcal{O}(F)), x$ is represented by the diagonal unitary matrix $U(x) \in M_{|x|}(\mathcal{O}(F))$, as in the proof of Lemma 2.1. Then $i_{*}(x)$ is represented by $U(x)+I-U(x)^{*} U(x) \in M_{|x|}(\mathcal{O}(G))$. But this is exactly the unitary matrix representing the element of $\mathbf{Z}^{G^{0}}$ given in the statement of the lemma.

Let $j_{F}: I_{F} \subseteq \mathcal{T} \mathcal{O}(F)$ be the inclusion. Then $i_{*} \circ j_{F *}: K_{0}\left(I_{F}\right) \rightarrow K_{0}(\mathcal{T O}(G))$ is easily computed to be

$$
i_{*} \circ j_{F *}\left[\varepsilon_{u, F}\right]=\left[\varepsilon_{u, G}\right]+\sum\left\{\left[e e^{*}\right] \mid e \in o^{-1}(u) \cap G^{1} \backslash F^{1}\right\} .
$$

We omit the proof.

Theorem 2.3. Let $G_{0}$ and $G_{1}$ be finitely generated abelian groups with $G_{1}$ free. Then there is an irreducible noncycle graph $E$ with $K_{*} \mathcal{O}(E) \cong\left(G_{0}, G_{1}\right)$. If rank $G_{0}$ 
$\neq \operatorname{rank} G_{1}$, then $E$ can be chosen along with a chain of subgraphs $\left(F_{n}\right)_{n \geq 0}$ so that conditions (a) and (b) are satisfied.

Proof. Case (i): $\operatorname{rank} G_{0}=\operatorname{rank} G_{1}$. The existence of such a graph $E$ is known, e.g., from [15]. Since we will need a specific graph with this property below, we describe it here. Suppose that

$$
\begin{aligned}
G_{0} & =\mathbf{Z}^{\ell} \oplus \mathbf{Z} /\left(n_{1}\right) \oplus \ldots \oplus \mathbf{Z} /\left(n_{k}\right), \\
G_{1} & =\mathbf{Z}^{\ell},
\end{aligned}
$$

where $k, \ell \geq 0$, and $n_{i} \geq 2$. We construct a graph $F$ with $k+\ell+1$ vertices $v_{1}, \ldots$, $v_{\ell}, z, w_{1}, \ldots, w_{k}$, by including edges as follows:

$$
\begin{aligned}
v_{i} & \longrightarrow\left\{v_{1}, \ldots, v_{\ell}, z\right\}, \\
z & \stackrel{n_{1}}{\longrightarrow} w_{1}, \\
w_{i} & \stackrel{n_{i+1}}{\longrightarrow} w_{i+1}, \quad 1 \leq i<k, \\
w_{k} & \longrightarrow\left\{v_{1}, \ldots, v_{\ell}, z\right\},
\end{aligned}
$$

and an additional loop at each vertex. It is clear that the graph $F$ is irreducible (and even primitive). Thus, with the vertices used in the order listed above, the incidence matrix of the graph is

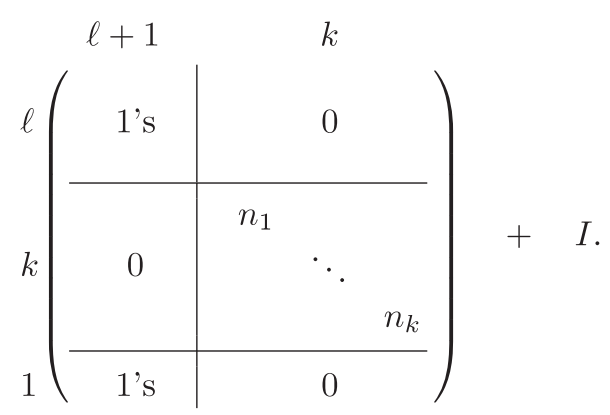

(If $k=0$ we use the sum of an $(\ell+1) \times(\ell+1)$ matrix of 1 's with the identity.) If $x \in \mathbf{Z}^{F^{0}}$ represents an element of $K_{1} \mathcal{O}(F)$, we obtain the following relations:

$$
\begin{aligned}
\sum_{i=1}^{\ell} x\left(v_{i}\right)+x\left(w_{k}\right) & =0, \text { from } v_{i} \text { or } z \\
x(z) & =0, \text { from } w_{1} \\
x\left(w_{i}\right) & =0, i<k, \text { from } w_{i+1} .
\end{aligned}
$$

It follows that $K_{1} \mathcal{O}(F) \cong \mathbf{Z}^{\ell}$, with basis $x_{1}, \ldots, x_{\ell}$, where

$$
x_{i}(s)=\left\{\begin{aligned}
1 & \text { if } s=v_{i} \\
-1 & \text { if } s=w_{k} \\
0 & \text { else }
\end{aligned}\right.
$$




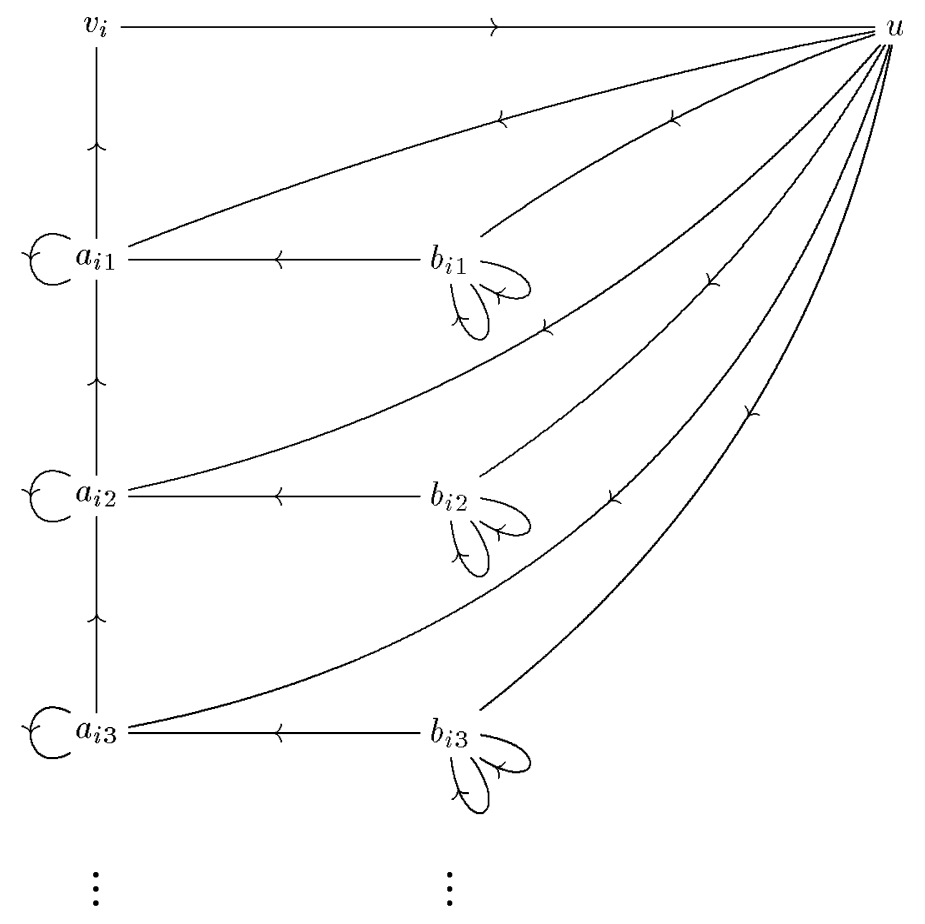

FIGURE 1. $\operatorname{rank} K_{1}>\operatorname{rank} K_{0}$

$K_{0} \mathcal{O}(F)$ is generated by the classes of the vertices, with relations

$$
\begin{aligned}
\sum_{i=1}^{\ell}\left[v_{i}\right]+[z] & =0, \text { from } v_{i} \text { or } w_{k} \\
n_{i}\left[w_{i}\right] & =0,1 \leq i \leq k, \text { from } z, w_{1}, \ldots, w_{k-1} .
\end{aligned}
$$

It follows that $K_{0} \mathcal{O}(F) \cong \mathbf{Z}^{\ell} \oplus \mathbf{Z} /\left(n_{1}\right) \oplus \ldots \oplus \mathbf{Z} /\left(n_{k}\right)$, with the cyclic subgroups generated by $\left[v_{1}\right], \ldots,\left[v_{\ell}\right],\left[w_{1}\right], \ldots,\left[w_{k}\right]$.

Case (ii): $\operatorname{rank} G_{0}<\operatorname{rank} G_{1}$. Let $G_{1} \cong \mathbf{Z}^{\ell-1}$ and $G_{0} \cong \mathbf{Z}^{\ell-p-1} \oplus \mathbf{Z} /\left(n_{1}\right) \oplus \ldots \oplus$ $\mathbf{Z} /\left(n_{k}\right)$, where $0<p<\ell$ and $\ell>1$. We build a larger graph, $E$, from $F$ as follows. Include a vertex $u$, and for $1 \leq i \leq p+1$ include the graph shown in Figure 1 .

For $n \geq 0$ we let $F_{n}$ be the subgraph of $E$ with

$$
\begin{aligned}
& F_{n}^{0}=F^{0} \cup\{u\} \cup\left\{a_{i j}, b_{i j} \mid 1 \leq i \leq p+1,1 \leq j \leq n+1\right\}, \\
& F_{n}^{1}=o^{-1}\left(F_{n}^{0}\right) \cap t^{-1}\left(F_{n}^{0}\right) .
\end{aligned}
$$

We will let $\mathcal{T} \mathcal{O}\left(F_{n}\right)$ denote $\mathcal{T} \mathcal{O}\left(F_{n}, F_{n}^{0} \backslash\{u\}\right)$. We compute $K_{*} \mathcal{O}\left(F_{n}\right)$. Let $x \in \mathbf{Z}^{F_{n}^{0}}$ represent an element of $K_{1} \mathcal{O}\left(F_{n}\right)$. Note that the relations (k1) and (k2) still hold ((k1) from the vertex $z$ ). For $1 \leq i \leq p+1$, consideration of the vertex $v_{i}$ gives

$$
\sum_{j=1}^{\ell} x\left(v_{j}\right)+x\left(w_{k}\right)+x\left(a_{i 1}\right)=0 .
$$


Combined with (k1), we find that

$$
x\left(a_{i 1}\right)=0,1 \leq i \leq p+1 .
$$

Consideration of the vertex $u$ gives

$$
x(u)=\sum_{i=1}^{p+1} x\left(v_{i}\right) .
$$

Finally, consideration of the remaining vertices shows that

$$
\begin{aligned}
& x\left(a_{i j}\right)=0, \\
& x\left(b_{i j}\right)=-x(u), \quad 1 \leq i \leq p+1,1 \leq j \leq n+1 .
\end{aligned}
$$

It follows from $(\mathrm{k} 1),(\mathrm{k} 2),(\mathrm{k} 6),(\mathrm{k} 7)$, and $(\mathrm{k} 8)$ that $K_{1} \mathcal{O}\left(F_{n}\right) \cong \mathbf{Z}^{\ell}$, with the same generators as for $K_{1} \mathcal{O}(F)$. Hence $(\mathrm{k} 7)$ implies that $K_{1} \mathcal{T} \mathcal{O}\left(F_{n}\right) \cong \mathbf{Z}^{\ell-1}$. Moreover it is clear that the inclusion $\mathcal{T O}\left(F_{n}\right) \subseteq \mathcal{T} \mathcal{O}\left(F_{n+1}\right)$ induces an isomorphism in $K_{1}$. Hence $K_{1} \mathcal{O}(E) \cong \mathbf{Z}^{\ell-1}$.

It also follows from $(\mathrm{k} 7)$ that $\partial$ is onto, so that $K_{0} \mathcal{T} \mathcal{O}\left(F_{n}\right) \cong K_{0} \mathcal{O}\left(F_{n}\right)$. To compute $K_{0} \mathcal{O}\left(F_{n}\right)$, notice that $(\mathrm{k} 3)$ and $(\mathrm{k} 4)$ still hold $\left((\mathrm{k} 3)\right.$ from the vertex $w_{k}$; or if $k=0$, from the vertex $z$ ). For $1 \leq j \leq p+1$, consideration of the vertex $v_{i}$ gives

$$
[u]+\sum_{j=1}^{\ell}\left[v_{j}\right]+[z]=0,
$$

and hence

$$
[u]=0 .
$$

Consideration of the vertices $a_{i j}$ shows that

$$
\begin{aligned}
{\left[v_{i}\right]=0, } & 1 \leq i \leq p+1, \\
{\left[a_{i j}\right] } & =0, \quad 1 \leq i \leq p+1,0 \leq j \leq n .
\end{aligned}
$$

Finally, consideration of the vertices $b_{i j}$ shows that $\left[b_{i j}\right]=2\left[b_{i j}\right]+\left[a_{i j}\right]$, and hence

$$
\left[b_{i j}\right]=-\left[a_{i j}\right], \quad 1 \leq i \leq p+1,0 \leq j \leq n+1 .
$$

It follows from (k3), (k4), (k9), (k10), and (k11) that $K_{0} \mathcal{O}\left(F_{n}\right) \cong \mathbf{Z}^{\ell} \oplus \mathbf{Z} /\left(n_{1}\right) \oplus$ $\ldots \oplus \mathbf{Z} /\left(n_{k}\right)$, with basis for the free summand given by

$$
\left[v_{p+2}\right], \ldots,\left[v_{\ell}\right],\left[a_{1, n+1}\right], \ldots,\left[a_{p+1, n+1}\right] .
$$

Thus the map $i_{*}: K_{0} \mathcal{T} \mathcal{O}\left(F_{n}\right) \rightarrow K_{0} \mathcal{T} \mathcal{O}\left(F_{n+1}\right)$ kills the last $p+1$ of the basis elements. Thus $K_{0} \mathcal{O}(E)=\lim _{\rightarrow} K_{0} \mathcal{T} \mathcal{O}\left(F_{n}\right) \cong \mathbf{Z}^{\ell-p-1} \oplus \mathbf{Z} /\left(n_{1}\right) \oplus \ldots \oplus \mathbf{Z} /\left(n_{k}\right)$.

Case (iii): $\operatorname{rank} G_{0}>\operatorname{rank} G_{1}$. Let $G_{1} \cong \mathbf{Z}^{\ell-p}$ and $G_{0} \cong \mathbf{Z}^{\ell} \oplus \mathbf{Z} /\left(n_{1}\right) \oplus \ldots \oplus \mathbf{Z} /\left(n_{k}\right)$, where $0<p \leq \ell$ and $\ell \geq 1$. We build a larger graph, $E$, from $F$ by attaching to each $v_{i}, 1 \leq i \leq p$, the graph shown in Figure 2 .

For $n \geq 0$ we let $F_{n}$ be the subgraph of $E$ given by

$$
\begin{aligned}
& F_{n}^{0}=F^{0} \cup\left\{u_{i} \mid 1 \leq i \leq p\right\} \cup\left\{a_{i j}, b_{i j} \mid 1 \leq i \leq p, 1 \leq j \leq n+2\right\}, \\
& F_{n}^{1}=o^{-1}\left(F_{n}^{0}\right) \cap t^{-1}\left(F_{n}^{0}\right),
\end{aligned}
$$




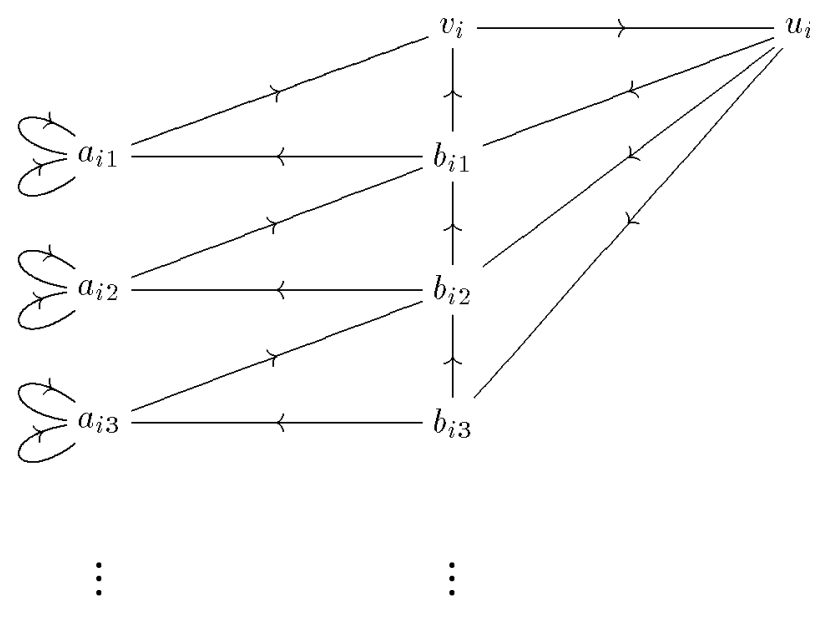

FIGURE 2. $\operatorname{rank} K_{1}<\operatorname{rank} K_{0}$

and we let $\mathcal{T} \mathcal{O}\left(F_{n}\right)$ denote $\mathcal{T} \mathcal{O}\left(F_{n}, F_{n}^{0} \backslash\left\{u_{i} \mid 1 \leq i \leq p\right\}\right)$. We first compute $K_{1} \mathcal{O}\left(F_{n}\right)$. Let $x \in \mathbf{Z}^{F_{n}^{0}}$ represent an element of $K_{1} \mathcal{O}\left(F_{n}\right)$. We still have (k1) and (k2), as in Case (ii). Consideration of vertex $a_{i j}$ gives

$$
x\left(a_{i j}\right)+x\left(b_{i j}\right)=0, \quad 1 \leq i \leq p, 1 \leq j \leq n+2 .
$$

Consideration of vertex $v_{i}, 1 \leq i \leq p$, gives

$$
\sum_{j=1}^{\ell} x\left(v_{j}\right)+x\left(w_{k}\right)+x\left(a_{i j}\right)+x\left(b_{i j}\right)=0,
$$

which is already contained in $(\mathrm{k} 1)$ and $(\mathrm{k} 12)$. Consideration of vertex $u_{i}$ gives

$$
x\left(u_{i}\right)=x\left(v_{i}\right), \quad 1 \leq i \leq p .
$$

Finally, consideration of vertex $b_{i j}$ gives

$$
x\left(b_{i j}\right)=x\left(u_{i}\right), \quad 1 \leq i \leq p, 0 \leq j \leq n+2
$$

(with a slightly different computation in the case $j=n+2$ ). It follows from (k3), $(\mathrm{k} 4),(\mathrm{k} 12),(\mathrm{k} 13)$, and $(\mathrm{k} 14)$ that $K_{1} \mathcal{O}\left(F_{n}\right) \cong \mathbf{Z}^{\ell}$. Moreover (k13) implies that $\partial$ is onto, so that $K_{1} \mathcal{T} \mathcal{O}\left(F_{n}\right) \cong \mathbf{Z}^{\ell-p}$, with basis $x_{p+1}, \ldots, x_{\ell}$ (where the $x_{i}$ are as defined in Case (ii)). It follows that $\mathcal{T O}\left(F_{n}\right) \subseteq \mathcal{T} \mathcal{O}\left(F_{n+1}\right)$ induces an isomorphism in $K_{1}$, so that $K_{1} \mathcal{O}(E) \cong \mathbf{Z}^{\ell-p}$.

Since $\partial$ is onto, $K_{0} \mathcal{T} \mathcal{O}\left(F_{n}\right) \cong K_{0} \mathcal{O}\left(F_{n}\right)$. To compute $K_{0} \mathcal{O}\left(F_{n}\right)$, notice that (k3) and (k4) still hold, as in Case (ii). Consideration of vertex $v_{i}$ gives

$$
\sum_{j=1}^{\ell}\left[v_{j}\right]+[z]+\left[u_{i}\right]=0
$$

and hence, from (k3),

$$
\left[u_{i}\right]=0, \quad 1 \leq i \leq p
$$




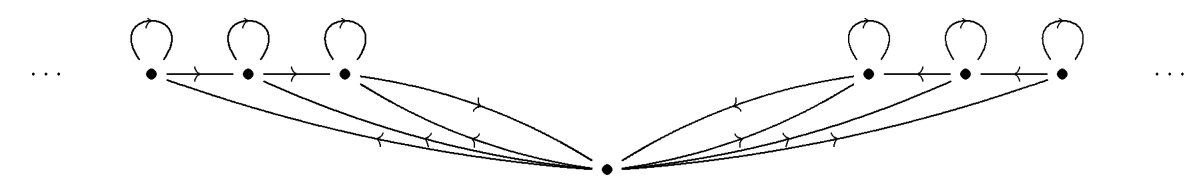

Figure $3 . K_{*} \mathcal{O}(E) \cong(0, \mathbf{Z})$

Consideration of vertex $a_{i j}$ gives

$$
\begin{aligned}
& {\left[a_{i 1}\right]=-\left[v_{i}\right], \quad 1 \leq i \leq p,} \\
& {\left[a_{i j}\right]=-\left[b_{i, j-1}\right], \quad 1 \leq i \leq p, 1 \leq j \leq n+2 .}
\end{aligned}
$$

Consideration of the vertices $b_{i j}$ inductively in $j$ gives

$$
\left[b_{i j}\right]=0, \quad 1 \leq i \leq p, 1 \leq j \leq n+2 .
$$

It follows from $(\mathrm{k} 3),(\mathrm{k} 4),(\mathrm{k} 15),(\mathrm{k} 16)$, and $(\mathrm{k} 17)$ that $K_{0} \mathcal{O}\left(F_{n}\right) \cong K_{0} \mathcal{O}(F)$, with the same generators and relations. Hence $\mathcal{T O}\left(F_{n}\right) \subseteq \mathcal{T} \mathcal{O}\left(F_{n+1}\right)$ induces an isomorphism in $K_{0}$, so that $K_{0} \mathcal{O}(E) \cong K_{0} \mathcal{O}(F)$.

Finally, we claim that in Cases (ii) and (iii) the graph $E$ and the chain of subgraphs $\left(F_{n}\right)_{n \geq 0}$ satisfy conditions (a) and (b). Conditions (a) and (b1) are clear. For (b2), in Case (ii) we identify $a_{i j}$ and $b_{i j}$ with $a_{i 1}$ and $b_{i 1}$, respectively, in the obvious way, for $j \geq 2$. In Case (iii) we identify $a_{i j}$ and $b_{i j}$ with $a_{i 2}$ and $b_{i 2}$, respectively, in the obvious way, for $j \geq 3$.

Remark 2.4. In the case where $G_{0}$ is also torsion-free, the graph $E$ can be simplified. We show in Figure 3 a graph $E$ for which $K_{0} \mathcal{O}(E) \cong 0$ and $K_{1} \mathcal{O}(E) \cong \mathbf{Z}$. (Neubüser, in [11, Proposition 3.17], has given a row-finite graph having the same $C^{*}$-algebra.) If the graph is expanded to have $\ell+1$ 'pieces' joined at the one vertex having infinite valence, a graph is obtained for which $K_{*} \cong\left(0, \mathbf{Z}^{\ell}\right)$. We leave it to the interested reader to check the details.

\section{Semiprojectivity}

In this section we prove the main theorem of the paper, Theorem 3.12.

Theorem 3.1. Let $E$ be an infinite directed graph with finite subgraphs $F_{0} \subseteq F_{1} \subseteq$ $\ldots \subseteq E$ such that $\bigcup F_{n}=E$. Let $D:=\left\{u \in E^{0} \mid o^{-1}(u)\right.$ is infinite $\}$. Suppose that conditions (a) and (b) from Section 1 hold. Then $\mathcal{O}(E)$ is semiprojective.

We will prove several lemmas before proving Theorem 3.1. Lemmas 3.2, 3.3, and 3.6 provide the main approximation arguments used in the proof of Theorem 3.1. Lemma 3.2 is a variation of a standard approximation result. See, e.g., [1, 2.23].

Lemma 3.2. There exist $\delta>0$ and a map $x \mapsto y \equiv y(x)$, defined on all elements of a $C^{*}$-algebra for which $\left\|x x^{*} x-x\right\|<\delta$, having the following properties:

(i) $y$ is a partial isometry.

(ii) $y \in C^{*}(x)$.

(iii) $y-x$ lies in the ideal of $C^{*}(x)$ generated by $x x^{*} x-x$.

(iv) $\|y-x\| \rightarrow 0$ as $\left\|x x^{*} x-x\right\| \rightarrow 0$.

(v) If $p$ is a multiplier projection with $p x=x$ (respectively, $p x=0, x p=x$, or $x p=0)$, then the same holds for $p$ and $y$. 
Proof. Letting $f(t)=t^{-1 / 2} \chi_{(1 / 2, \infty)}(t)$ for $t \geq 0$, then $y(x)=x f\left(x^{*} x\right)$.

The next lemma uses Lemma 3.2 to 'straighten' a family of approximate generators that extend a given set of generators from one subgraph to the next. It is arranged so that we may use it on four separate occasions in the proof of Theorem 3.1 .

Lemma 3.3. Let $F \subseteq G$ be an inclusion of finite directed irreducible graphs, neither a cycle. Let $D \subseteq F^{0}$, and suppose that (d1) $o^{-1}\left(F^{0} \backslash D\right) \subseteq F^{1}$, (d2) $o^{-1}\left(F^{0}\right) \cap t^{-1}\left(F^{0}\right) \subseteq F^{1}$.

Let $M$ be a directed set, and for $\mu \in M$ let $B_{\mu}$ be a $C^{*}$-algebra with ideal $I_{\mu}$. Suppose that for each $\mu \in M$ we are given elements $\left\{c_{\mu}(e) \mid e \in G^{1}\right\} \subseteq B_{\mu}$ such that properties (d3)-(d5) (below) hold:

(d3) $\left\{c_{\mu}(e) \mid e \in F^{1}\right\}$ satisfy condition $(\mathcal{O})$.

For $u \in F^{0}$, let $\xi_{\mu}(u)=c_{\mu}(e)^{*} c_{\mu}(e)$ for any e $\in F^{1} \cap t^{-1}(u)$. Let $p_{\mu}=\sum\left\{\xi_{\mu}(u) \mid\right.$ $\left.u \in F^{0}\right\}$, and for $u \in D$, let $q_{\mu}(u)=\sum\left\{c_{\mu}(e) c_{\mu}(e)^{*} \mid e \in F^{1} \cap o^{-1}(u)\right\}$.

(d4) For $e \in G^{1} \backslash F^{1}, c_{\mu}(e)$ satisfies:

$$
c_{\mu}(e)= \begin{cases}\left(1-p_{\mu}\right) c_{\mu}(e) \xi_{\mu}(t(e)) & \text { if } o(e) \notin D \text { and } t(e) \in F^{0} ; \\ \left(1-p_{\mu}\right) c_{\mu}(e)\left(1-p_{\mu}\right) & \text { if } o(e) \notin D \text { and } t(e) \notin F^{0} ; \\ \left(\xi_{\mu}(u)-q_{\mu}(u)\right) c_{\mu}(e)\left(1-p_{\mu}\right) & \text { if } o(e)=u \in D .\end{cases}
$$

(d5) $\left\{c_{\mu}(e) \mid e \in G^{1}\right\}$ satisfy condition $(\mathcal{O})$ asymptotically, in the sense that the relevant quantities belong to $I_{\mu}$, and tend to 0 as $\mu \rightarrow \infty$.

Then there exists $\mu_{0}$ such that for all $\mu \geq \mu_{0}$ there are elements $\left\{a_{\mu}(e) \mid e \in\right.$ $\left.G^{1}\right\} \subseteq B_{\mu}$ for which properties (d6)-(d9) (below) hold:

(d6) $\left\{a_{\mu}(e) \mid e \in G^{1}\right\}$ satisfy condition $(\mathcal{O})$.

(d7) Property (d4) above holds for $\left\{a_{\mu}(e) \mid e \in G^{1} \backslash F^{1}\right\}$.

(d8) $a_{\mu}(e)=c_{\mu}(e)$ for $e \in F^{1}$.

(d9) For all $e \in G^{1}, a_{\mu}(e)-c_{\mu}(e) \in I_{\mu}$ and $\left\|a_{\mu}(e)-c_{\mu}(e)\right\| \rightarrow 0$ as $\mu \rightarrow \infty$.

Proof. Let $G^{1} \backslash F^{1} \backslash o^{-1}(D)=\left\{e_{1}, e_{2}, \ldots, e_{m}\right\}$. For all large enough $\mu, c_{\mu}\left(e_{1}\right)$ satisfies the hypotheses of Lemma 3.2. For such a $\mu$, let $b_{\mu}\left(e_{1}\right)$ be the partial isometry obtained from $c_{\mu}\left(e_{1}\right)$ as in Lemma 3.2. Inductively, for all large enough $\mu$, we can let $b_{\mu}\left(e_{j}\right)$ be the partial isometry obtained from $\left[1-\sum_{i<j} b_{\mu}\left(e_{i}\right) b_{\mu}\left(e_{i}\right)^{*}\right] c_{\mu}\left(e_{j}\right)$ as in Lemma 3.2. Then $\left\{b_{\mu}\left(e_{1}\right), \ldots, b_{\mu}\left(e_{m}\right)\right\}$ are partial isometries whose final projections are pairwise orthogonal. For $u \in G^{0} \backslash F^{0}$, let

$$
b_{\mu}(u)=\sum\left\{b_{\mu}(e) b_{\mu}(e)^{*} \mid e \in o^{-1}(u)\right\} .
$$

Note that

$$
b_{\mu}(u) \leq 1-p_{\mu}
$$

by the first two lines of property $(\mathrm{d} 4)$.

We now consider $\left(G^{1} \backslash F^{1}\right) \cap o^{-1}(D)$. Fix $u \in D$. Let $\left(G^{1} \backslash F^{1}\right) \cap o^{-1}(u)=$ $\left\{f_{1}, \ldots, f_{n}\right\}$. As before, for all large enough $\mu$, let $b_{\mu}\left(f_{j}\right)$ be the partial isometry obtained from $\left[1-\sum_{i<j} b_{\mu}\left(f_{i}\right) b_{\mu}\left(f_{i}\right)^{*}\right] c_{\mu}\left(f_{j}\right)$ as in Lemma 3.2. Then $\left\{b_{\mu}\left(f_{1}\right), \ldots\right.$, $\left.b_{\mu}\left(f_{n}\right)\right\}$ are partial isometries whose final projections are pairwise orthogonal. 
Notice that property (d4) holds for $\left\{b_{\mu}(e) \mid e \in G^{1} \backslash F^{1}\right\}$, by Lemma 3.2 and (d10). Notice also that for all $e \in G^{1} \backslash F^{1}, b_{\mu}(e)-c_{\mu}(e) \in I_{\mu}$ and $\left\|b_{\mu}(e)-c_{\mu}(e)\right\| \rightarrow 0$ as $\mu \rightarrow \infty$, by (d5).

Now, for $e \in G^{1} \backslash F^{1}$, and all large enough $\mu$, if $t(e) \notin F^{0}$, then $b_{\mu}(e)^{*} b_{\mu}(e)-$ $b_{\mu}(t(e))$ is a small element of $I_{\mu}$ (by the asymptotic version of condition (o6)). Similarly, if $t(e) \in F^{0}$, then $b_{\mu}(e)^{*} b_{\mu}(e)-\xi_{\mu}(t(e))$ is a small element of $I_{\mu}$. It follows from Lemma 3.2 that for all large enough $\mu$, there is a partial isometry $d_{\mu}(e) \in B_{\mu}$ such that

$$
\begin{aligned}
d_{\mu}(e)^{*} d_{\mu}(e) & = \begin{cases}b_{\mu}(t(e)) & \text { if } t(e) \notin F^{0}, \\
\xi_{\mu}(t(e)) & \text { if } t(e) \in F^{0}\end{cases} \\
d_{\mu}(e) d_{\mu}(e)^{*} & =b_{\mu}(e)^{*} b_{\mu}(e) ; \\
d_{\mu}(e)-b_{\mu}(e)^{*} b_{\mu}(e) & \in I_{\mu} ; \\
\left\|d_{\mu}(e)-b_{\mu}(e)^{*} b_{\mu}(e)\right\| & \rightarrow 0 \text { as } \mu \rightarrow \infty .
\end{aligned}
$$

Set

$$
a_{\mu}(e)= \begin{cases}b_{\mu}(e) d_{\mu}(e) & \text { if } e \in G^{1} \backslash F^{1}, \\ c_{\mu}(e) & \text { if } e \in F^{1}\end{cases}
$$

Notation 3.4. Let $B$ be a $C^{*}$-algebra, $J$ an ideal in $B$, and $\Lambda$ a quasi-central approximate unit for $J$. If $x=x(h)$ and $y=y(h)$ are elements of $B$ depending on a variable $h \in \Lambda$, we will write $x \approx y$ to mean that $x-y \in J$ for all $h$, and $\lim _{h}\|x-y\|=0$.

Remark 3.5. Let $B$ be a $C^{*}$-algebra, $J$ an ideal in $B$, and $\Lambda$ a quasi-central approximate unit for $J$. For $h \in \Lambda$, let $k=k(h)=\left(1-h^{2}\right)^{1 / 2}$. Then for any $x \in B$, $k x \approx x k$, and for any $y \in J, k y \approx 0$.

The next lemma is the key technique used in the proof of Theorem 3.1. It allows us to construct a partial isometry from a given partial isometry and a more suitable element which, however, only becomes a partial isometry after dividing by an ideal.

Lemma 3.6. Let $B$ be a $C^{*}$-algebra and let $J$ be an ideal in $B$. Let $a, b \in B$ be such that $a$ is a partial isometry and $b+J$ is a partial isometry $($ in $B / J)$. Suppose that one of the following cases holds:

(i) $a^{*} b, a^{*} a-b^{*} b \in J$;

(ii) $a^{*} b, a b^{*} \in J$, and $a^{*} a+J \sim b^{*} b+J$ in $B / J$.

Let $r \in B$ be such that

$$
r^{*} r-a^{*} a, r r^{*}-b^{*} b \in J
$$

where we let $r=a^{*} a$ in case (i). Let $\Lambda$ be a quasi-central approximate unit for $J$. Let $h$ and $k$ be as in Remark 3.5. For $h \in \Lambda$ define $w=w(h) \in B$ by

$$
w= \begin{cases}h a+k b, & \text { in case (i); } \\ h^{2} a+k^{2} b+h k\left(a r^{*}+b r\right), & \text { in case (ii). }\end{cases}
$$

Then $w w^{*} w \approx w$. Moreover we have the following asymptotic formulas for $w^{*} w$ and $w w^{*}$ :

$$
w^{*} w \approx \begin{cases}a^{*} a, & \text { in case }(i) ; \\ h^{2} a^{*} a+k^{2} b^{*} b+h k\left(r+r^{*}\right), & \text { in case }(i i) ;\end{cases}
$$


and

$$
w w^{*} \approx h^{2} a a^{*}+k^{2} b b^{*}+h k\left(b r a^{*}+a r^{*} b^{*}\right) .
$$

Proof. We first consider case (i). Using Remark 3.5 we find

$$
\begin{aligned}
w^{*} w & =a^{*} h^{2} a+b^{*} k^{2} b+2 \operatorname{Re}\left(a^{*} h k b\right) \\
& \approx h^{2} a^{*} a+k^{2} b^{*} b+2 \operatorname{Re}\left(h k a^{*} b\right) \\
& \approx h^{2} a^{*} a+k^{2} a^{*} a+0 \\
& =a^{*} a .
\end{aligned}
$$

This establishes the first asymptotic formula. Moreover, since $a^{*} a$ is a projection, we have $\left(w^{*} w\right)^{2} \approx w^{*} w$, and hence $\left(w w^{*} w-w\right)^{*}\left(w w^{*} w-w\right)=\left(w^{*} w\right)^{3}-2\left(w^{*} w\right)^{2}+$ $w^{*} w \approx 0$. Thus $w w^{*} w \approx w$. For the second asymptotic formula, we have

$$
\begin{aligned}
w w^{*} & =h a a^{*} h+k b b^{*} k+2 \operatorname{Re}\left(h a b^{*} k\right) \\
& \approx h^{2} a a^{*}+k^{2} b b^{*}+h k\left(b r a^{*}+a r^{*} b^{*}\right) .
\end{aligned}
$$

We now treat case (ii). First note that, along with those explicitly mentioned in the statement of the lemma, the following elements (and their adjoints) belong to $J$ : $a r, b r^{*}, r a^{*} a-r, b^{*} b r-r$, and $r^{2}$ (the last because $r-r r^{*} r \in J$ and $\left(r r^{*} r\right)^{2}=r a^{*} a b^{*} b r \in J$, so that $\left.r^{2}=\left[r r^{*} r+\left(r-r r^{*} r\right)\right]^{2} \in J\right)$. In particular, by Remark 3.5, if $x$ is any of these elements, then $k x \approx 0$. Using Remark 3.5 repeatedly gives

$$
\begin{aligned}
w^{*} w= & {\left[a^{*} h^{2}+b^{*} k^{2}+\left(r a^{*}+r^{*} b^{*}\right) h k\right]\left[h^{2} a+k^{2} b+h k\left(a r^{*}+b r\right)\right] } \\
\approx & h^{4} a^{*} a+h^{3} k a^{*} a r^{*}+k^{4} b^{*} b+h k^{3} b^{*} b r \\
& +h^{3} k r a^{*} a+h k^{3} r^{*} b^{*} b+h^{2} k^{2}\left(r a^{*} a r^{*}+r^{*} b^{*} b r\right) \\
\approx & h^{4} a^{*} a+h^{3} k r^{*}+k^{4} b^{*} b+h k^{3} r+h^{3} k r+h k^{3} r^{*}+h^{2} k^{2}\left(b^{*} b+a^{*} a\right) \\
= & \left(h^{4}+h^{2} k^{2}\right) a^{*} a+\left(k^{4}+h^{2} k^{2}\right) b^{*} b+\left(h^{3} k+h k^{3}\right)\left(r+r^{*}\right) \\
= & h^{2} a^{*} a+k^{2} b^{*} b+h k\left(r+r^{*}\right),
\end{aligned}
$$

establishing the first asymptotic formula. The second is proved in the same way, and we omit the details. Finally, applying similar computations to the first asymptotic formula gives $\left(w^{*} w\right)^{2} \approx w^{*} w$, and hence, as in case (i), $w w^{*} w \approx w$.

At this point we wish to briefly sketch the main idea of the proof of Theorem 3.1. The first step is to lift an initial Toeplitz algebra, $\mathcal{T} \mathcal{O}\left(F_{2}\right)$, modulo some ideal. Then we extend that lift to a $*$-homomorphism on all of $\mathcal{O}(E)$. However, we will inductively extend the lifting from $F_{0}$ to $F_{1}$, etc. In order to use Lemma 3.6 for this we have to arrange the initial extension so as to 'miss' $F_{1} \backslash F_{0}$. Lemma 3.9 below is our way of making this arrangement.

Lemma 3.7. Let $F$ be an irreducible graph and not a cycle. Let $D \subseteq F^{0}$ and $a \in F^{0}$. Then $\mathcal{T} \mathcal{O}\left(F, D^{c}\right)$ contains an infinite family of partial isometries with pairwise orthogonal final projections less than a, and initial projections equal to a. 
Proof. Choose two distinct cycles at $a$. Let $s$ and $t$ denote the corresponding products of edges. Then $s, t \in \mathcal{T} \mathcal{O}\left(F, D^{c}\right)$ satisfy

$$
\begin{aligned}
s^{*} s=t^{*} t & =a, \\
s s^{*}, t t^{*} & \leq a, \\
s^{*} t & =0 .
\end{aligned}
$$

It follows that $\left\{s^{i} t \mid i=0,1,2, \ldots\right\}$ are partial isometries with initial projections all equal to $a$, and pairwise orthogonal final projections less than $a$.

Lemma 3.8. Let $F, D$, and $a$ be as in Lemma 3.7. Then $\mathcal{T} \mathcal{O}\left(F, D^{c}\right)$ contains a family $\Omega$ of pairwise orthogonal subprojections of a such that every vertex in $F^{0}$ is equivalent to infinitely many elements of $\Omega$.

Proof. By Lemma 3.7 there is an infinite family of partial isometries in $\mathcal{T} \mathcal{O}\left(F, D^{c}\right)$ with pairwise orthogonal final projections less than $a$, and initial projections equal to $a$. Divide these into pairwise disjoint infinite subfamilies $\left\{\Delta_{u} \mid u \in F^{0}\right\}$. For each $u \in F^{0}$ choose a path from $a$ to $u$; let $r_{u}$ denote the corresponding product of edges. Then $r_{u}^{*} r_{u}=u$ and $r_{u} r_{u}^{*} \leq a$. Let $\Omega=\bigcup_{u \in F^{0}}\left\{s r_{u} r_{u}^{*} s^{*} \mid s \in \Delta_{u}\right\}$.

Lemma 3.9. Let $E$ and $\left(F_{n}\right)_{n \geq 0}$ satisfy conditions (a) and (b) (and hence also (a6), (b3), and (b4), by Remarks 1.2). For each $n \geq 2$ choose $a_{n} \in F_{n}^{0} \backslash F_{n-1}^{0}$, and a map $e_{n}: D \rightarrow F_{n}^{1} \backslash F_{n-1}^{1}$ with $e_{n, u} \in o^{-1}(u)$ for $u \in D$. (Note that $e_{n, u}$ exists by condition (a6) of Remarks 1.1.) Then there is a $*$-homomorphism $\phi_{n}: \mathcal{O}(E) \rightarrow \mathcal{T} \mathcal{O}\left(F_{n}\right)$ such that

$$
\begin{array}{llll}
\text { (c1) } & \phi_{n}(u) \sim u & \text { for } u \in E^{0} \\
\text { (c2) } & \phi_{n}(u) \leq a_{n} & \text { for } u \in E^{0} \backslash D ; \\
\text { (c3) } & \phi_{n}(u)=u & \text { for } u \in D ; \\
\text { (c4) } & \phi_{n}(f) \phi_{n}(f)^{*} \leq e_{n, u} e_{n, u}^{*} & \text { for } & f \in o^{-1}(u), u \in D .
\end{array}
$$

Proof. Fix $n \geq 2$. For this proof we will omit the subscript $n$ (so that $F=F_{n}$, $a=a_{n}, e_{u}=e_{n, u}$, etc.). By Lemma 3.8 there is a family $\Omega$ of pairwise orthogonal subprojections of $a$ in $\mathcal{T O}(F)$ such that each vertex in $F^{0}$ is equivalent (in $\mathcal{T O}(F)$ ) to infinitely many elements of $\Omega$. For each $u \in D$ choose a path in $F$ from $t\left(e_{u}\right)$ to $a$; let $\xi_{u}$ denote the corresponding product of edges. Let $\Omega_{u}=\left\{e_{u} \xi_{u} \omega \xi_{u}^{*} e_{u}^{*} \mid \omega \in \Omega\right\}$. Then $\Omega_{u} \subseteq \mathcal{T O}(F)$ is a pairwise orthogonal family of subprojections of $e_{u} e_{u}^{*}$ such that each vertex in $F^{0}$ is equivalent (in $\mathcal{T O}(F)$ ) to infinitely many elements of $\Omega_{u}$.

We first define $\phi$ on $E^{0}$, then on $E^{1}$. For $u \in D$ we let $\phi(u)=u$. For $u \notin D$ choose $v_{u} \in F_{0}^{0} \backslash D$ as in (b2). (Let $v_{u}=u$ if $u \in F_{0}^{0}$.) Thus there is a graph isomorphism $\gamma_{u}: o^{-1}(u) \rightarrow o^{-1}\left(v_{u}\right)$ such that for all $e \in o^{-1}(u),[t(e)]=\left[t\left(\gamma_{u}(e)\right)\right]$ in $K_{0}(\mathcal{O}(E))$. Choose $\phi: E^{0} \backslash D \rightarrow \Omega$ so that $\phi(u) \sim v_{u}$ in $\mathcal{T} \mathcal{O}(F)$, and so that $\phi$ is one-to-one. Let $\alpha_{u} \in \mathcal{T} \mathcal{O}(F)$ with

$$
\begin{aligned}
& \alpha_{u}^{*} \alpha_{u}=\phi(u), \\
& \alpha_{u} \alpha_{u}^{*}=v_{u} .
\end{aligned}
$$

(For $u \in D$ define $\alpha_{u}=v_{u}=u$.)

We claim that $u \sim v_{u}$ in $\mathcal{O}(E)$, for all $u \in E^{0}$. This is immediate for $u \in D$. For $u \in E^{0} \backslash D$ consider $e \in o^{-1}(u)$. We have $[t(e)]=\left[t\left(\gamma_{u}(e)\right)\right]$. By [5], $t(e) \sim t\left(\gamma_{u}(e)\right)$ 
in $\mathcal{O}(E)$. Hence

$$
\begin{aligned}
u & =\sum\left\{e e^{*} \mid e \in o^{-1}(u)\right\} \\
& \sim \sum\left\{\gamma_{u}(e) \gamma_{u}(e)^{*} \mid e \in o^{-1}(u)\right\}
\end{aligned}
$$

establishing the claim. Now if $e \in o^{-1}(u)$ for $u \notin D$, then

$$
\left[t\left(\gamma_{u}(e)\right)\right]=[t(e)]=\left[v_{t(e)}\right] .
$$

By $(\mathrm{b} 4), t\left(\gamma_{u}(e)\right) \sim v_{t(e)}$ in $\mathcal{T O}(F)$. Hence $t\left(\gamma_{u}(e)\right) \sim \phi(t(e))$ in $\mathcal{T} \mathcal{O}(F)$. Let $\beta_{e} \in \mathcal{T} \mathcal{O}(F)$ with

$$
\begin{aligned}
& \beta_{e}^{*} \beta_{e}=\phi(t(e)), \\
& \beta_{e} \beta_{e}^{*}=t\left(\gamma_{u}(e)\right) .
\end{aligned}
$$

We define $\phi$ on $E^{1} \backslash o^{-1}(D)$ by

$$
\phi(e)=\alpha_{u}^{*} \gamma_{u}(e) \beta_{e}, \text { for } e \in o^{-1}(u), u \notin D .
$$

We compute for $u \notin D$ :

$$
\begin{aligned}
\phi(e)^{*} \phi(e) & =\beta_{e}^{*} \gamma_{u}(e)^{*} \alpha_{u} \alpha_{u}^{*} \gamma_{u}(e) \beta_{e} \\
& =\beta_{e}^{*} \gamma_{u}(e)^{*} v_{u} \gamma_{u}(e) \beta_{e} \\
& =\beta_{e}^{*} t\left(\gamma_{u}(e)\right) \beta_{e} \\
& =\phi(t(e)) \\
\phi(e) \phi(e)^{*} & =\alpha_{u}^{*} \gamma_{u}(e) \beta_{e} \beta_{e}^{*} \gamma_{u}(e)^{*} \alpha_{u} \\
& =\alpha_{u}^{*} \gamma_{u}(e) \gamma_{u}(e)^{*} \alpha_{u}, \\
\sum_{e \in o^{-1}(u)} \phi(e) \phi(e)^{*} & =\alpha_{u}^{*}\left(\sum_{e \in o^{-1}(u)} \gamma_{u}(e) \gamma_{u}(e)^{*}\right) \alpha_{u} \\
& =\alpha_{u}^{*} v_{u} \alpha_{u} \\
& =\phi(u)
\end{aligned}
$$

Finally, let $u \in D$. Choose a one-to-one map $\epsilon_{u}: o^{-1}(u) \rightarrow \Omega_{u}$ such that $\epsilon_{u}(e) \sim \phi(t(e))$ in $\mathcal{T} \mathcal{O}(F)$ for $e \in o^{-1}(u)$. For $e \in o^{-1}(u)$ let $\phi(e) \in \mathcal{T} \mathcal{O}(F)$ satisfy

$$
\begin{aligned}
& \phi(e)^{*} \phi(e)=\phi(t(e)), \\
& \phi(e) \phi(e)^{*}=\epsilon_{u}(e) .
\end{aligned}
$$

It is now easy to see that $\phi: \mathcal{O}(E) \rightarrow \mathcal{T} \mathcal{O}(F)$ satisfies the requirements of the lemma.

Lemma 3.11 below is a necessary technical device for the main inductive step in the proof of Theorem 3.1. We let $\mathcal{U}(A)$ denote the unitary group of a unital $C^{*}$-algebra $A$.

Lemma 3.10. Let $F$ be a finite irreducible graph which is not a cycle. Let $D \subseteq F^{0}$, and let $\mathcal{T} \mathcal{O}(F)$ denote $\mathcal{T} \mathcal{O}\left(F, D^{c}\right)$. Then for any vertex $a \in F^{0}$, the map

$$
\mathcal{U}(a \cdot \mathcal{T} \mathcal{O}(F) \cdot a) \rightarrow K_{1}(\mathcal{T} \mathcal{O}(F))
$$

is onto. 
Proof. Let $x=(x(e))_{e \in F^{1}} \in \mathbf{Z}^{\left|F^{1}\right|}$ represent an element of $K_{1}(\mathcal{T} \mathcal{O}(F))$. Then $x(e)=0$ whenever $o(e) \in D$. It follows that the element $U_{0}(x) \in M_{|x|}(\mathcal{T O}(F))$ constructed in Lemma 2.1 is unitary, and $\left[U_{0}(x)\right]$ represents $x$ in $K_{1}(\mathcal{T} \mathcal{O}(F))$.

Now let $a \in F_{0}$. It follows from Lemma 3.7 that there are partial isometries $s_{1}$, $s_{2}, \ldots \in \mathcal{T} \mathcal{O}(F)$ with initial projections equal to $a$ and pairwise orthogonal final projections less than $a$. For each $u \in F^{0}$ choose a path from $a$ to $u$, and let $r_{u}$ denote the corresponding product of edges. Then $r_{u}^{*} r_{u}=u$ and $r_{u} r_{u}^{*} \leq a$. Let $F^{0}=\left\{u_{1}, u_{2}, \ldots, u_{k}\right\}$, and for $n \geq 1$ set

$$
t_{n}=\sum_{i=1}^{k} s_{(n-1) k+i} r_{u_{i}} .
$$

Then $t_{1}, t_{2}, \ldots$ are isometries in $\mathcal{T} \mathcal{O}(F)$ with orthogonal ranges dominated by $a$. Now $t=\left(t_{1}, t_{2}, \ldots, t_{k}\right)$ is a partial isometry with

$$
t^{*} t=1_{M_{|x|} \mathcal{T} \mathcal{O}(F)} \text { and } t t^{*} \leq a .
$$

Then $t U_{0}(x) t^{*}+a-t t^{*} \in \mathcal{U}(a \cdot \mathcal{T O}(F) \cdot a)$ is equivalent to $U_{0}(x)$.

Lemma 3.11. Let $B$ be a $C^{*}$-algebra, let $\mathcal{L}$ be a directed family of ideals of $B$ with closure $I$, and suppose that $B / I$ is simple and purely infinite. Let $p \in B$ be a projection such that $\mathcal{U}(p B p) \rightarrow K_{1}(B / I)$ is onto. Let $x \in B$ and $J_{1} \in \mathcal{L}$ be such that

$$
x^{*} x-p, x x^{*}-p \in J_{1} .
$$

Then there is $J_{2} \in \mathcal{L}$ with $J_{1} \subseteq J_{2}$, and there is $y \in B$ such that

$$
y^{*} y=y y^{*}=p \quad \text { and } \quad x-y \in J_{2} .
$$

Proof. Replacing $B$ by $p B p$ and $\mathcal{L}$ by $p \mathcal{L} p$, we may assume that $B$ is unital and that $x+J_{1}$ is unitary. By the assumption on $K$-theory we may choose $y_{1} \in \mathcal{U}(B)$ such that $\left[y_{1}+I\right]=[x+I]$ in $K_{1}(B / I)$. Then $\left[y_{1}^{*} x+I\right]=0$. By $[5], y_{1}^{*} x+I$ is in the connected component of the identy in $\mathcal{U}(B / I)$, and hence is a product of exponentials. Since exponentials lift to $B$ there is $y_{2} \in \mathcal{U}(B)$ with $y_{1}^{*} x-y_{2} \in I$. Then $x-y_{1} y_{2} \in I$, and so there is $J_{2} \in \mathcal{L}, J_{1} \subseteq J_{2}$, such that $\left\|x-y_{1} y_{2}+J_{2}\right\|<$ 1. Now $y_{2}^{*} y_{1}^{*} x+J_{2}$ lies in the unit ball centered at the identity, and hence is an exponential. Thus there is $y_{3} \in \mathcal{U}(B)$ with $y_{2}^{*} y_{1}^{*} x-y_{3} \in J_{2}$. We may take $y=y_{1} y_{2} y_{3}$.

Proof of Theorem 3.1. Let $B$ be a $C^{*}$-algebra, let $\mathcal{L}$ be a directed family of ideals of $B$ with closure $I$, and suppose that $B / I$ is isomorphic to $\mathcal{O}(E)$ (we will identify $B / I$ with $\mathcal{O}(E))$. For any $J \in \mathcal{L}$, we will use $\pi: B / J \rightarrow B / I$ to denote the quotient map (the context should make it clear what the domain of $\pi$ is). If $J^{\prime} \subseteq J$ are two elements of $\mathcal{L}$, we will write $\pi_{J}: B / J^{\prime} \rightarrow B / J$, or $\pi_{n}: B / J_{i} \rightarrow B / J_{n}$ if $J_{i} \subseteq J_{n}$, and if $x^{\prime} \in J^{\prime}, x \in J$, we will write $x^{\prime} x$ for $\pi_{J}\left(x^{\prime}\right) x$. We also recall the *-homomorphisms $\phi_{n}: \mathcal{O}(E) \rightarrow \mathcal{T} \mathcal{O}\left(F_{n}\right)$ provided by Lemma 3.9.

Note that Lemma 3.3 is applicable in the case where the smaller graph is empty. Thus we can find $J_{0} \in \mathcal{L}$ and a $*$-homomorphism $\theta_{0}: \mathcal{T O}\left(F_{2}\right) \rightarrow B / J_{0}$ such that

$\left(2_{0}\right) \quad \pi \circ \theta_{0}=\mathrm{id}_{\mathcal{T} \mathcal{O}\left(F_{2}\right)}$.

For $u \in F_{0}^{0} \backslash D$, recall from the proof of Lemma 3.9 the element $\alpha_{2, u} \in \mathcal{T} \mathcal{O}\left(F_{2}\right)$ with $\alpha_{2, u}^{*} \alpha_{2, u}=\phi_{2}(u)$ and $\alpha_{2, u} \alpha_{2, u}^{*}=v_{u}=u$. Define $\psi_{0}: \mathcal{O}(E) \rightarrow B / J_{0}$ by:

$$
\left.\psi_{0}\right|_{\mathcal{T} \mathcal{O}\left(F_{0}\right)}=\left.\theta_{0}\right|_{\mathcal{T} \mathcal{O}\left(F_{0}\right)}
$$


$\left(4_{00}\right)$

$$
\begin{array}{ll}
\psi_{0}(e)=\theta_{0}\left(\phi_{2}(e)\right) & \text { if } e \in E^{1} \backslash F_{0}^{1} \text { and } t(e) \notin F_{0}^{0} ; \\
\psi_{0}(e)=\theta_{0}\left(\phi_{2}(e) \alpha_{2, t(e)}^{*}\right), & \text { if } e \in E^{1} \backslash F_{0}^{1} \text { and } t(e) \in F_{0}^{0} .
\end{array}
$$

It is easy to check that $\psi_{0}$ is a $*$-homomorphism. We remark that $\left(4_{00}\right)$ implies the following:

$$
\begin{array}{ll}
\psi_{0}(u)=\theta_{0}\left(\phi_{2}(u)\right) & \text { if } u \in E^{0} \backslash F_{0}^{0} . \\
\psi_{0}\left(e e^{*}\right)=\theta_{0}\left(\phi_{2}\left(e e^{*}\right)\right) & \text { if } e \in E^{1} \backslash F_{0}^{1} \text { and } o(e) \in D .
\end{array}
$$

Suppose inductively that we have constructed for $j<n$ the following:

$$
\begin{aligned}
& J_{j} \in \mathcal{L}, \\
& \theta_{j}: \mathcal{T} \mathcal{O}\left(F_{j+2}\right) \rightarrow B / J_{j}, \text { a } * \text {-homomorphism, } \\
& \psi_{j}: \mathcal{O}(E) \rightarrow B / J_{0}, \text { a } * \text {-homomorphism }
\end{aligned}
$$

such that

$$
\begin{array}{lll}
\left(1_{j}\right) & J_{j-1} \subseteq J_{j}, \\
\left(2_{j}\right) & \pi \circ \theta_{j}=\operatorname{id}_{\mathcal{T} \mathcal{O}\left(F_{j+2}\right)}, \\
\left(3_{j}\right) & \left.\pi_{j} \circ \psi_{j}\right|_{\mathcal{T} \mathcal{O}\left(F_{j}\right)}=\left.\theta_{j}\right|_{\mathcal{T} \mathcal{O}\left(F_{j}\right)}, & \\
\left(4_{j}\right) & \pi_{j} \circ \psi_{j}(u)=\theta_{j}\left(\phi_{j+2}(u)\right) \quad \text { if } u \in E^{0} \backslash F_{j}^{0}, \\
& \pi_{j} \circ \psi_{j}\left(e e^{*}\right)=\theta_{j}\left(\phi_{j+2}\left(e e^{*}\right)\right) \quad \text { if } e \in E^{1} \backslash F_{j}^{1} \text { and } o(e) \in D, \\
\left(5_{j}\right) \quad & \left.\theta_{j}\right|_{\mathcal{T} \mathcal{O}\left(F_{j+1}\right)}=\pi_{j} \circ \theta_{j-1}, \\
\left(6_{j}\right) \quad & \left.\psi_{j}\right|_{\mathcal{T} \mathcal{O}\left(F_{j-1}\right)}=\left.\psi_{j-1}\right|_{\mathcal{T} \mathcal{O}\left(F_{j-1}\right)} .
\end{array}
$$$$
\left.\left(5_{j}\right) \quad \theta_{j}\right|_{\mathcal{T} \mathcal{O}\left(F_{j+1}\right)}=\pi_{j} \circ \theta_{j-1},
$$

We will construct $J_{n}, \theta_{n}, \psi_{n}$ satisfying the above for $j=n$. Then $\psi=\lim _{n} \psi_{n}$ will establish the theorem. First we will define $\theta_{n}$. For this we need to consider edges in $F_{n+2}^{1} \backslash F_{n+1}^{1}$.

For $e \in F_{n+2}^{1} \backslash F_{n+1}^{1}$ choose $\sigma(e) \in B / J_{n-1}$ such that

$$
\left(7_{n-1}\right) \quad \pi(\sigma(e))=e .
$$

Let

$$
\begin{aligned}
p & =\sum\left\{\theta_{n-1}(u) \mid u \in F_{n+1}^{0}\right\}, \\
q(u) & =\sum\left\{\theta_{n-1}(e) \theta_{n-1}(e)^{*} \mid e \in F_{n+1}^{1} \cap o^{-1}(u)\right\}, \text { for } u \in D .
\end{aligned}
$$

We will apply Lemma 3.3 with

$$
\begin{aligned}
F & =F_{n+1}, \\
G & =F_{n+2}, \\
M & =\left\{J \in \mathcal{L} \mid J \supseteq J_{n-1}\right\}, \\
B_{J} & =B / J, \\
I_{J} & =I / J, \\
c_{J}(e) & =\pi_{J}(c(e)),
\end{aligned}
$$

where

$$
c(e)=\theta_{n-1}(e) \text { if } e \in F_{n+1}^{1},
$$


and for $e \in F_{n+2}^{1} \backslash F_{n+1}^{1}$,

$$
c(e)= \begin{cases}(1-p) \sigma(e) \theta_{n-1}(t(e)) & \text { if } o(e) \notin D \text { and } t(e) \in F_{n+1}^{0}, \\ (1-p) \sigma(e)(1-p) & \text { if } o(e) \notin D \text { and } t(e) \notin F_{n+1}^{0}, \\ \left(\theta_{n-1}(u)-q(u)\right) \sigma(e)(1-p) & \text { if } o(e)=u \in D\end{cases}
$$

(Note that $p_{J}$ and $q_{J}(u)$ in Lemma 3.3 are given by $\pi_{J}(p)$ and $\pi_{J}(q(u))$.) Then properties (d1)-(d4) of Lemma 3.3 are immediate, and (d5) follows from $\left(2_{n-1}\right)$ and $\left(7_{n-1}\right)$ above. By Lemma 3.3 there exist $J_{n}^{\prime} \in \mathcal{L}$ and a $*$-homomorphism $\theta_{n}^{\prime}: \mathcal{T} \mathcal{O}\left(F_{n+2}\right) \rightarrow B / J_{n}^{\prime}$ satisfying $\left(1_{n}\right),\left(2_{n}\right)$, and $\left(5_{n}\right)$.

The construction of $\psi_{n}$ proceeds in two stages. First we extend $\psi_{n-1}$ from $F_{n-1}$ to $F_{n}$ satisfying $\left(3_{n}\right)$; then we extend it from $F_{n}$ to $E$ satisfying $\left(4_{n}\right)$. Each stage will require another application of Lemma 3.3.

For $u \in F_{n}^{0} \backslash F_{n-1}^{0}$ choose $r_{0}(u) \in B / J_{n}^{\prime}$ with

$$
\begin{aligned}
& r_{0}(u)^{*} r_{0}(u)=\pi_{n}^{\prime} \circ \psi_{n-1}(u), \\
& r_{0}(u) r_{0}(u)^{*}=\theta_{n}^{\prime}(u) .
\end{aligned}
$$

(Such elements exist because

$$
\begin{aligned}
\pi_{n}^{\prime} \circ \psi_{n-1}(u) & =\pi_{n}^{\prime} \circ \pi_{n-1} \circ \psi_{n-1}(u) \\
& =\pi_{n}^{\prime} \circ \theta_{n-1}\left(\phi_{n+1}(u)\right), \quad \text { by }\left(4_{n-1}\right), \\
& =\theta_{n}^{\prime}\left(\phi_{n+1}(u)\right), \quad \text { by }\left(5_{n}\right), \\
& \left.\sim \theta_{n}^{\prime}(u), \quad \text { by Lemma } 3.9,(\mathrm{c} 1) .\right)
\end{aligned}
$$

For $u \in F_{n-1}^{0}$ we set $r_{0}(u)=\pi_{n}^{\prime} \circ \psi_{n-1}(u)$. For $u \in F_{n}^{0} \backslash F_{n-1}^{0}$, let

$$
r(u)=\sum\left\{\theta_{n}^{\prime}(e) r_{0}(t(e)) \psi_{n-1}(e)^{*} \mid e \in F_{n}^{1} \cap o^{-1}(u)\right\},
$$

and set $r(u)=r_{0}(u)$ for $u \in F_{n-1}^{0}$. Then $(*)$ holds for $r(u)$ in place of $r_{0}(u)$. Let $u \in F_{n}^{0} \backslash F_{n-1}^{0}$. By $(*)$ for $r_{0}(u)$ and for $r(u)$, we have that $r_{0}(u)^{*} r(u)$ is a unitary on (the range of) $\pi_{n}^{\prime} \circ \psi_{n-1}(u)$. By condition (b3) and Lemmas 3.10 and 3.11, there exist $y(u) \in B / J_{0}$ and $J(u) \in \mathcal{L}$ with $J_{n}^{\prime} \subseteq J(u)$ such that

$$
\begin{aligned}
y(u)^{*} y(u) & =y(u) y(u)^{*}=\psi_{n-1}(u), \\
\pi_{n}^{\prime}(y(u)) & \equiv r_{0}(u)^{*} r(u) \quad(\bmod J(u)) .
\end{aligned}
$$

For $u \in F_{n-1}^{0}$ we let $y(u)=\psi_{n-1}(u)$. Now for any $e \in F_{n}^{1}$ we set

$$
\psi_{n-1}^{\prime}(e)=\psi_{n-1}(e) y(t(e)) .
$$

Then $\psi_{n-1}^{\prime}(e)$ is a partial isometry having the same initial and final projections as $\psi_{n-1}(e)$. Hence $\left\{\psi_{n-1}^{\prime}(e) \mid e \in F_{n}^{1}\right\}$ define a $*$-homomorphism of $\mathcal{T} \mathcal{O}\left(F_{n}\right)$. We note that

$$
\left.\psi_{n-1}^{\prime}\right|_{\mathcal{T O}\left(F_{n-1}\right)}=\left.\psi_{n-1}\right|_{\mathcal{T O}\left(F_{n-1}\right)} .
$$

Choose $J_{n} \in \mathcal{L}$ with $J(u) \subseteq J_{n}$ for all $u \in F_{n}^{0} \backslash F_{n-1}^{0}$. We now define $\theta_{n}$ : $\mathcal{T O}\left(F_{n+2}\right) \rightarrow B / J_{n}$ by

$$
\theta_{n}=\pi_{n} \circ \theta_{n}^{\prime} .
$$


Now let $u \in F_{n}^{0} \backslash F_{n-1}^{0}$ and $e \in o^{-1}(u)$. We compute:

$$
\begin{aligned}
\pi_{n}\left(r(u) \psi_{n-1}^{\prime}(e) \psi_{n-1}^{\prime}(e)^{*}\right) & =\pi_{n}\left(r(u) \psi_{n-1}(e) \psi_{n-1}(e)^{*}\right) \\
& =\pi_{n}\left(\theta_{n}^{\prime}(e) r_{0}(t(e)) \psi_{n-1}(e)^{*}\right), \text { by definition of } r(u), \\
& =\theta_{n}(e) r_{0}(t(e)) y(t(e)) \psi_{n-1}^{\prime}(e)^{*} \\
& =\theta_{n}(e) r(t(e)) \psi_{n-1}^{\prime}(e)^{*}
\end{aligned}
$$

Since

$$
r(u)=\sum_{e \in o^{-1}(u)} r(u) \psi_{n-1}^{\prime}(e) \psi_{n-1}^{\prime}(e)^{*}
$$

then

$$
\pi_{n}(r(u))=\sum_{e \in o^{-1}(u)} \theta_{n}(e) r(t(e)) \psi_{n-1}^{\prime}(e)^{*}
$$

For $u \in F_{n}^{0}$ and $e \in F_{n}^{1}$ choose arbitrarily $\widetilde{r}(u), \widetilde{\theta}_{n}(u), \widetilde{\theta}_{n}(e) \in B / J_{0}$ lifting $r(u)$, $\theta_{n}(u)$, and $\theta_{n}(e)$. Let $\Lambda$ be a quasi-central approximate unit for $J_{n}$, and let $h, k$ be as in Remark 3.5. For $e \in F_{n}^{1} \backslash F_{n-1}^{1}$, let $w(e)$ be defined as in Lemma 3.6, with $a=\psi_{n-1}^{\prime}(e), b=\widetilde{\theta}_{n}(e)$, and $r=\widetilde{r}(t(e))$. We will apply Lemma 3.3 with

$$
\begin{aligned}
F & =F_{n-1}, \\
G & =F_{n}, \\
M & =\Lambda, \\
B_{h} & =B / J_{0}, h \in \Lambda, \\
I_{h} & =J_{n} / J_{0}, h \in \Lambda, \\
c_{h}(e) & =\psi_{n-1}(e), \text { if } e \in F_{n-1}^{1},
\end{aligned}
$$

and for $e \in F_{n}^{1} \backslash F_{n-1}^{1}$,

$$
c_{h}(e)= \begin{cases}\left(1-p_{h}\right) w(e) \psi_{n-1}(t(e)) & \text { if } o(e) \notin D \text { and } t(e) \in F_{n-1}^{0}, \\ \left(1-p_{h}\right) w(e)\left(1-p_{h}\right) & \text { if } o(e) \notin D \text { and } t(e) \notin F_{n-1}^{0}, \\ \left(\psi_{n-1}(u)-q_{h}(u)\right) w(e)\left(1-p_{h}\right) & \text { if } o(e)=u \in D,\end{cases}
$$

where, as in the statement of Lemma 3.3, $p_{h}=\sum\left\{\psi_{n-1}(u) \mid u \in F_{n-1}^{0}\right\}$, and for $u \in D, q_{h}(u)=\sum\left\{\psi_{n-1}(e) \psi_{n-1}(e)^{*} \mid e \in F_{n-1}^{1} \cap o^{-1}(u)\right\}$. Then (d1)-(d4) of Lemma 3.3 hold by definition. To establish (d5) of Lemma 3.3, first note that by Remark 3.5, if $e \in F_{n}^{1} \backslash F_{n-1}^{1}$, then $c_{h}(e) \approx w(e)$.

We will check that condition $(\mathcal{O})$ holds asymptotically. It follows from Lemma 3.6 that $w(e) w(e)^{*} w(e) \approx w(e)$, so $c_{h}(e)$ is asymptotically a partial isometry. For $u \in F_{n}^{0} \backslash F_{n-1}^{0}$ and $e \in t^{-1}(u)$, Lemma 3.6 gives

$$
\begin{aligned}
w(e)^{*} w(e) & \approx h^{2} \psi_{n-1}(u)+k^{2} \widetilde{\theta}_{n}(e)^{*} \widetilde{\theta}_{n}(e)+2 h k \operatorname{Re}(\widetilde{r}(u)) \\
& \approx h^{2} \psi_{n-1}(u)+k^{2} \widetilde{\theta}_{n}(u)+2 h k \operatorname{Re}(\widetilde{r}(u)),
\end{aligned}
$$

which is independent of $e \in t^{-1}(u)$. Define

$$
c_{h}(u)=h^{2} \psi_{n-1}(u)+k^{2} \widetilde{\theta}_{n}(u)+2 h k \operatorname{Re}(\widetilde{r}(u)) .
$$

Then $c_{h}(u)$ is asymptotically a projection. This establishes (o1). 
For (o2), let $u, v \in F_{n}^{0}$ be distinct. If $u, v \in F_{n-1}^{0}$, then

$$
c_{h}(u) c_{h}(v)=\psi_{n-1}(u) \psi_{n-1}(v)=0 .
$$

If $u \in F_{n-1}^{0}$ and $v \notin F_{n-1}^{0}$,

$$
\begin{aligned}
c_{h}(u) c_{h}(v) & =\psi_{n-1}(u)\left[h^{2} \psi_{n-1}(v)+k^{2} \widetilde{\theta}_{n}(v)+2 h k \operatorname{Re}(\widetilde{r}(v))\right] \\
& \approx 0
\end{aligned}
$$

by Remark 3.5, since

$$
\begin{aligned}
\pi_{n} \circ \psi_{n-1}(u) & =\pi_{n} \circ \pi_{n-1} \circ \psi_{n-1}(u) \\
& =\pi_{n} \circ \theta_{n-1}\left(\phi_{n+1}(u)\right), \text { by }\left(5_{n-1}\right), \\
& =\theta_{n}\left(\phi_{n+1}(u)\right)
\end{aligned}
$$

is orthogonal to $\theta_{n}(v)$. The case where $u, v \notin F_{n-1}^{0}$ is similar.

Condition (o3) follows from the definition of $c_{h}(u)$, and Lemma 3.6.

For (o4) let $u \in D$ and $e \in F_{n}^{1} \cap o^{-1}(u)$. If $e \in F_{n-1}^{1}$, the condition is clear. If $e \notin F_{n-1}^{1}$, then

$$
\begin{aligned}
c_{h}(u) c_{h}(e) & \approx \psi_{n-1}(u)\left[h^{2} \psi_{n-1}^{\prime}(e)+k^{2} \widetilde{\theta}_{n}(e)+2 h k \operatorname{Re}\left(\widetilde{\theta}_{n}(e) \widetilde{r}(t(e)) \psi_{n-1}^{\prime}(e)^{*}\right)\right] \\
& \approx c_{h}(e)
\end{aligned}
$$

since $\psi_{n-1}(u) \psi_{n-1}^{\prime}(e)=\psi_{n-1}^{\prime}(e)$ and $\pi_{n}\left(\psi_{n-1}(u)\right) \theta_{n}(u) \theta_{n}(e)=\theta_{n}(e)$.

For (o5) let $u \in D$ and $e, f \in o^{-1}(u) \cap F_{n}^{1}$ with $e \neq f$. If $e, f \in F_{n-1}^{1}$, the condition is clear. If $e \in F_{n-1}^{1}$ and $f \notin F_{n-1}^{1}$, then

$$
c_{h}(e)^{*} c_{h}(f)=\left[q_{h}(u) c_{h}(e)\right]^{*}\left[\left(\psi_{n-1}(u)-q_{h}(u)\right) c_{h}(f)\right]=0 .
$$

If $e, f \notin F_{n-1}^{1}$, then $c_{h}(e)^{*} c_{h}(f) \approx 0$, since

$$
\begin{aligned}
\pi_{n}\left(\psi_{n-1}^{\prime}(e)^{*}\right) \theta_{n}(f) & =\pi_{n}\left(\psi_{n-1}(e) y(t(e))\right)^{*} \theta_{n}(f) \\
& =\pi_{n}(y(t(e)))^{*} \pi_{n} \circ \theta_{n-1}\left(\phi_{n+1}(e)\right)^{*} \theta_{n}(f) \\
& =\pi_{n}(y(t(e)))^{*} \theta_{n}\left(\phi_{n+1}(e)\right)^{*} \theta_{n}(f) \\
& =0 .
\end{aligned}
$$

For (o6) let $u \notin D$. If $u \in F_{n-1}^{0}$, the condition is clear. If $u \in F_{n}^{1} \backslash F_{n-1}^{1}$, then $o^{-1}(u) \subseteq F_{n}^{1} \backslash F_{n-1}^{1}$. We compute (notice the use of condition $(* *)$ ):

$$
\begin{aligned}
\sum_{e \in o^{-1}(u)} c_{h}(e) c_{h}(e)^{*} & \approx \sum_{e \in o^{-1}(u)} w(e) w(e)^{*} \\
& \approx \sum_{e \in o^{-1}(u)} h^{2} \psi_{n-1}^{\prime}(e) \psi_{n-1}^{\prime}(e)^{*}+k^{2} \widetilde{\theta}_{n}(e) \widetilde{\theta}_{n}(e)^{*} \\
& +2 h k \operatorname{Re}\left[\widetilde{\theta}_{n}(e) \widetilde{r}(t(e)) \psi_{n-1}^{\prime}(e)^{*}\right] \\
& \approx h^{2} \psi_{n-1}^{\prime}(u)+k^{2} \widetilde{\theta}_{n}(u)+2 h k \operatorname{Re}[\widetilde{r}(u)], \quad \text { by }(* *), \\
& =c_{h}(u) .
\end{aligned}
$$

It now follows from Lemma 3.3 that there are $\left\{\psi_{n}(e) \mid e \in F_{n}^{1}\right\} \subseteq B / J_{0}$ such that $\left(3_{n}\right)$ and $\left(6_{n}\right)$ hold, and $\psi_{n}: \mathcal{T O}\left(F_{n}\right) \rightarrow B / J_{0}$ is a $*$-homomorphism. Moreover, 
we may assume that

$$
\psi_{n}(u) \sim \psi_{n-1}(u), \quad u \in F_{n}^{0} \backslash F_{n-1}^{0} .
$$

The reason is that Lemma 3.6 (i) applies to the element

$$
\lambda_{u}=h \psi_{n-1}(u)+k \widetilde{r}(u),
$$

and so

$$
\begin{aligned}
& \lambda_{u}^{*} \lambda_{u} \approx \psi_{n-1}(u), \\
& \lambda_{u} \lambda_{u}^{*} \approx c_{h}(u) .
\end{aligned}
$$

Thus in the previous application of Lemma 3.3, we may choose $h$ large enough in $\Lambda$ so that Lemma 3.2 applies to $\psi_{n}(u) \lambda_{n} \psi_{n-1}(u)$, giving the desired equivalence.

It remains to define $\left\{\psi_{n}(e) \mid e \in E^{1} \backslash F_{n}^{1}\right\}$ so that $\left\{\psi_{n}(e) \mid e \in E^{1}\right\}$ satisfy $(\mathcal{O})$ and so that $\left(4_{n}\right)$ holds. We will extend the definition of $r(u)$ to all $u \in E^{0}$, and will define additional elements $s(e)$ for $e \in E^{1} \backslash F_{n}^{1}$ with $o(e) \in D$. Recall from the proof of Lemma 3.9 the elements $\alpha_{n, u} \in \mathcal{T} \mathcal{O}\left(F_{n}\right)$, for $u \in E^{0} \backslash F_{n}^{0}$, with

$$
\begin{aligned}
& \alpha_{n, u}^{*} \alpha_{n, u}=\phi_{n}(u), \\
& \alpha_{n, u} \alpha_{n, u}^{*}=v_{u} .
\end{aligned}
$$

We let

$$
\begin{aligned}
& r(u)=\theta_{n}\left(\alpha_{n+2, u}^{*} \alpha_{n+1, u}\right), \quad u \in E^{0} \backslash F_{n}^{0}, \\
& s(e)=\theta_{n}\left(\phi_{n+2}(e) \alpha_{n+2, t(e)}^{*} \alpha_{n+1, t(e)} \phi_{n+1}(e)^{*}\right), \quad e \in E^{1} \backslash F_{n}^{1} \text { and } o(e) \in D .
\end{aligned}
$$

Then we have

$$
\begin{aligned}
r(u)^{*} r(u) & =\theta_{n}\left(\phi_{n+1}(u)\right)=\pi_{n}\left(\psi_{n-1}(u)\right), \\
r(u) r(u)^{*} & =\theta_{n}\left(\phi_{n+2}(u)\right), \\
s(e)^{*} s(e) & =\theta_{n}\left(\phi_{n+1}\left(e e^{*}\right)\right)=\pi_{n}\left(\psi_{n-1}\left(e e^{*}\right)\right), \\
s(e) s(e)^{*} & =\theta_{n}\left(\phi_{n+2}\left(e e^{*}\right)\right) .
\end{aligned}
$$

Let $\widetilde{r}(u), \widetilde{s}(e) \in B / J_{0}$ with $\pi_{n}(\widetilde{r}(u))=r(u), \pi_{n}(\widetilde{s}(e))=s(e)$.

Now we proceed with the definition of $\psi_{n}$. Suppose inductively that for some $i>n$ we have extended $\left.\psi_{n}\right|_{\mathcal{T} \mathcal{O}\left(F_{i-2}\right)}$ to a $*$-homomorphism $\psi_{n}: \mathcal{T O}\left(F_{i-1}\right) \rightarrow B / J_{0}$ satisfying $\left(4_{n}\right)$ on $F_{i-1} \backslash F_{n}$, and such that for $u \in F_{i-1}^{0} \backslash F_{i-2}^{0}$ there exists $\lambda_{u} \in B / J_{0}$ with

$$
\begin{aligned}
\lambda_{u}^{*} \lambda_{u} & =\psi_{n-1}(u), \\
\lambda_{u} \lambda_{u}^{*} & =\psi_{n}(u) .
\end{aligned}
$$

Let $h_{i} \in \Lambda$ and $k_{i}=\left(1-h_{i}{ }^{2}\right)^{-1 / 2}$ be as in Remark 3.5. We define

$$
w_{u}=h_{i} \psi_{n-1}(u)+k_{i} \widetilde{r}(u), \quad u \in F_{i}^{0} \backslash F_{i-1}^{0},
$$

and

$$
w_{e}=h_{i} \psi_{n-1}\left(e e^{*}\right)+k_{i} \widetilde{s}(e), \quad e \in\left(F_{i}^{1} \backslash F_{i-1}^{1}\right) \cap o^{-1}(D) .
$$

It follows from Lemma 3.6 (i) that

$$
w_{u}^{*} w_{u} \approx \psi_{n-1}(u), \quad u \in F_{i}^{0} \backslash F_{i-1}^{0},
$$

and

$$
w_{e}^{*} w_{e} \approx \psi_{n-1}\left(e e^{*}\right), \quad e \in\left(F_{i}^{1} \backslash F_{i-1}^{1}\right) \cap o^{-1}(D) .
$$


For $e \in F_{i}^{1} \backslash F_{i-1}^{1}$, we set

$$
\eta(e)= \begin{cases}w_{o(e)} \psi_{n-1}(e) \lambda_{t(e)}^{*} & \text { if } o(e) \notin D \text { and } t(e) \in F_{i-1}^{0}, \\ w_{o(e)} \psi_{n-1}(e) w_{t(e)}^{*} & \text { if } o(e) \notin D \text { and } t(e) \notin F_{i-1}^{0}, \\ w_{e} \psi_{n-1}(e) w_{t(e)}^{*} & \text { if } o(e) \in D .\end{cases}
$$

We will apply Lemma 3.3 with

$$
\begin{aligned}
F & =F_{i-1}, \\
G & =F_{i}, \\
M & =\Lambda, \\
B_{h_{i}} & =B / J_{0}, h_{i} \in \Lambda, \\
I_{h_{i}} & =J_{n} / J_{0}, h_{i} \in \Lambda, \\
c_{h_{i}}(e) & =\psi_{n}(e), \text { if } e \in F_{i-1}^{1},
\end{aligned}
$$

and for $e \in F_{i}^{1} \backslash F_{i-1}^{1}$,

$$
c_{h_{i}}(e)= \begin{cases}\left(1-p_{h_{i}}\right) \eta(e) \psi_{n}(t(e)) & \text { if } o(e) \notin D \text { and } t(e) \in F_{i-1}^{0}, \\ \left(1-p_{h_{i}}\right) \eta(e)\left(1-p_{h_{i}}\right) & \text { if } o(e) \notin D \text { and } t(e) \notin F_{i-1}^{0}, \\ \left(\psi_{n}(u)-q_{h_{i}}(u)\right) \eta(e)\left(1-p_{h_{i}}\right) & \text { if } o(e)=u \in D\end{cases}
$$

(where

$$
\begin{aligned}
p_{h_{i}} & =\sum\left\{\psi_{n}(u) \mid u \in F_{n-1}^{0}\right\}, \text { and } \\
q_{h_{i}}(u) & =\sum\left\{\psi_{n}(e) \psi_{n}(e)^{*} \mid e \in F_{n-1}^{1} \cap o^{-1}(u)\right\}, \quad u \in D,
\end{aligned}
$$

as in Lemma 3.3.) Then (d1)-(d4) of Lemma 3.3 hold by definition. Moreover, for $e \in F_{i}^{1} \backslash F_{i-1}^{1}$ we have

$$
c_{h_{i}}(e) \approx \eta(e)
$$

as can be seen from the facts:

$$
\begin{array}{ll}
\pi_{n}\left(w_{u}\right)=r(u), \quad u \in F_{i}^{0} \backslash F_{i-1}^{0}, \\
\pi_{n}\left(w_{e}\right)=s(e), \quad e \in\left(F_{i}^{1} \backslash F_{i-1}^{1}\right) \cap o^{-1}(u), \quad u \in D .
\end{array}
$$

We will now check the asymptotic condition $(\mathcal{O})$ for $\left\{c_{h_{i}}(e) \mid i \in F_{i}^{1} \backslash F_{i-1}^{1}\right\}$. For (o1) we note that if $u \in F_{i}^{0} \backslash F_{i-1}^{0}$ and $t(e)=u$, then

$$
\begin{aligned}
c_{h_{i}}(e)^{*} c_{h_{i}}(e) & \approx \eta(e)^{*} \eta(e) \\
& =w_{t(e)} \psi_{n-1}(e)^{*} w_{o(e)}^{*} w_{o(e)} \psi_{n-1}(e) w_{t(e)}^{*} \\
& \approx w_{t(e)} \psi_{n-1}(e)^{*} \psi_{n-1}(o(e)) \psi_{n-1}(e) w_{t(e)}^{*} \\
& =w_{t(e)} \psi_{n-1}(t(e)) w_{t(e)}^{*} \\
& \approx w_{t(e)} w_{t(e)}^{*}
\end{aligned}
$$

is independent of $e \in t^{-1}(u)$. Thus we may set

$$
c_{h_{i}}(u)=w_{u} w_{u}^{*}
$$


For (o2) let $u, v \in F_{i}^{0}$ be distinct. If $u, v \in F_{i-1}^{0}$, then $c_{h_{i}}(u) c_{h_{i}}(v)=0$ by the inductive hypothesis. If $u \in F_{i-1}^{0}$ and $v \notin F_{i-1}^{0}$, then

$$
\begin{aligned}
c_{h_{i}}(u) c_{h_{i}}(v) & \approx c_{h_{i}}(u) c_{h_{i}}(e)^{*} c_{h_{i}}(e), \text { for any } e \in t^{-1}(v), \\
& =0,
\end{aligned}
$$

by definition of $c_{h_{i}}(e)$. If $u, v \notin F_{i-1}^{0}$, then we have

$$
\begin{aligned}
w_{u}^{*} w_{v} & \approx h_{i}{ }^{2} \psi_{n-1}(u) \psi_{n-1}(v)+h_{i} k_{i}\left(\psi_{n-1}(u) \widetilde{r}(v)+\widetilde{r}(u)^{*} \psi_{n-1}(v)\right)+k_{i}{ }^{2} \widetilde{r}(u)^{*} \widetilde{r}(v) \\
& \approx 0
\end{aligned}
$$

since $\psi_{n-1}(u) \widetilde{r}(v), \psi_{n-1}(v) \widetilde{r}(u)$, and $\widetilde{r}(u)^{*} \widetilde{r}(v) \in J_{n}$. Hence

$$
c_{h_{i}}(u) c_{h_{i}}(v)=w_{u} w_{u}^{*} w_{v} w_{v}^{*} \approx 0 .
$$

Condition (o3) is immediate from the definition of $c_{h_{i}}(u)$.

For (o4), let $u \in D$ and $e \in F_{i}^{1} \cap o^{-1}(u)$. If $e \in F_{i-1}^{1}$, the condition follows from the inductive hypothesis. Suppose that $e \notin F_{i-1}^{1}$. Then

$$
\begin{aligned}
\psi_{n}(u) w_{e} & =\psi_{n}(u)\left(h_{i} \psi_{n-1}\left(e e^{*}\right)+k_{i} \widetilde{s}(e)\right) \\
& \approx h_{i} \psi_{n-1}\left(e e^{*}\right)+k_{i} \widetilde{s}(e)
\end{aligned}
$$

since $\phi_{\ell}\left(e e^{*}\right) \leq e_{\ell, u} e_{\ell, u}^{*} \leq u$ for all $\ell$,

$$
=w_{e}
$$

Since $u \in D, c_{h_{i}}(u)=\psi_{n}(u)$. Then

$$
\begin{aligned}
c_{h_{i}}(u) c_{h_{i}}(e) & \approx \psi_{n}(u) \eta(e) \\
& \approx \psi_{n}(u) w_{e} \psi_{n-1}(e) w_{t(e)}^{*} \\
& \approx w_{e} \psi_{n-1}(e) w_{t(e)}^{*} \\
& =\eta(e) \\
& \approx c_{h_{i}}(e)
\end{aligned}
$$

For (o5), let $u \in D$ and $e, f \in F_{i}^{1} \cap o^{-1}(u)$ with $e \neq f$. If $e, f \in F_{i-1}^{1}$, the condition follows from the inductive hypothesis. If $e \in F_{i-1}^{1}$ and $f \notin F_{i-1}^{1}$, the condition follows from the definition of $c_{h_{i}}(f)$. If $e, f \notin F_{i-1}^{1}$, then

$$
\begin{aligned}
c_{h_{i}}(e)^{*} c_{h_{i}}(f) & \approx \eta(e)^{*} \eta(f) \\
& =w_{t(e)} \psi_{n-1}(e)^{*} w_{e}^{*} w_{f} \psi_{n-1}(f) w_{t(f)}^{*} \\
& \approx 0
\end{aligned}
$$

since

$$
\begin{aligned}
w_{e}^{*} w_{f} & \approx\left(h_{i} \psi_{n-1}\left(e e^{*}\right)+k_{i} \widetilde{s}(e)\right)^{*}\left(h_{i} \psi_{n-1}\left(f f^{*}\right)+k_{i} \widetilde{s}(f)\right) \\
& \approx h_{i} k_{i}\left(\psi_{n-1}\left(e e^{*}\right) \widetilde{s}(f)+\widetilde{s}(e)^{*} \psi_{n-1}\left(f f^{*}\right)\right)+k_{i}{ }^{2} \widetilde{s}(e)^{*} \widetilde{s}(f) \\
& \approx 0
\end{aligned}
$$

by Remark 3.5. 
For (o6), let $u \in F_{i}^{0} \backslash D$. If $u \in F_{i-1}^{0}$, the condition follows from the inductive hypothesis. If $u \notin F_{i-1}^{0}$, then

$$
\begin{aligned}
& \sum\left\{c_{h_{i}}(e) c_{h_{i}}(e)^{*} \mid e \in o^{-1}(u)\right\} \approx \sum\left\{\eta(e) \eta(e)^{*} \mid e \in o^{-1}(u)\right\} \\
&= \sum\left\{w_{u} \psi_{n-1}(e) w_{t(e)}^{*} w_{t(e)} \psi_{n-1}(e)^{*} w_{u}^{*} \mid e \in o^{-1}(u) \backslash t^{-1}\left(F_{i-1}^{0}\right)\right\} \\
&+\sum\left\{w_{u} \psi_{n-1}(e) \lambda_{t(e)} \lambda_{t(e)}^{*} \psi_{n-1}(e)^{*} w_{u}^{*} \mid e \in o^{-1}(u) \cap t^{-1}\left(F_{i-1}^{0}\right)\right\} \\
& \approx \sum\left\{w_{u} \psi_{n-1}(e) \psi_{n-1}(t(e)) \psi_{n-1}(e)^{*} w_{u}^{*} \mid e \in o^{-1}(u)\right\} \\
&= w_{u} \psi_{n-1}(u) w_{u}^{*} \\
& \approx w_{u} w_{u}^{*} \\
&= c_{h_{i}}(u) .
\end{aligned}
$$

It now follows from Lemma 3.3 that there are $\left\{\psi_{n}(e) \mid e \in F_{i}^{1} \backslash F_{i-1}^{1}\right\}$ such that $\left\{\psi_{n}(e) \mid e \in F_{i}^{1}\right\}$ satisfy $(\mathcal{O})$. We now check $\left(4_{n}\right)$ on $F_{i} \backslash F_{i-1}$. For $u \in F_{i}^{0} \backslash F_{i-1}^{0}$,

$$
\begin{aligned}
\pi_{n} \circ \psi_{n}(u) & =\pi_{n}\left(c_{h_{i}}(u)\right) \\
& =\pi_{n}\left(w_{u} w_{u}^{*}\right) \\
& =r(u) r(u)^{*} \\
& =\theta_{n}\left(\phi_{n+2}(u)\right) .
\end{aligned}
$$

For $e \in F_{i}^{1} \backslash F_{i-1}^{1}$ with $o(e)=u \in D$,

$$
\begin{aligned}
\pi_{n} \circ \psi_{n}\left(e e^{*}\right) & =\pi_{n}\left(c_{h_{i}}(e) c_{h_{i}}(e)^{*}\right) \\
& =\pi_{n}\left(\eta(e) \eta(e)^{*}\right) \\
& =\pi_{n}\left(w_{e} \psi_{n-1}(e) w_{t(e)}^{*}\right) \pi_{n}\left(w_{e} \psi_{n-1}(e) w_{t(e)}^{*}\right)^{*} \\
& =s(e) \pi_{n}\left(\psi_{n-1}(e)\right) r(t(e))^{*} r(t(e)) \psi_{n-1}(e)^{*} s(e)^{*} \\
& =s(e) \pi_{n}\left(\psi_{n-1}(e)\right) \pi_{n}\left(\psi_{n-1}(e)\right)^{*} s(e)^{*} \\
& =\theta_{n}\left(\phi_{n+2}\left(e e^{*}\right)\right) .
\end{aligned}
$$

Finally, we note that for $u \in F_{i}^{0} \backslash F_{i-1}^{0}$, the element $w_{u}$ satisfies

$$
\begin{aligned}
w_{u}^{*} w_{u} & \approx \psi_{n-1}(u), \\
w_{u} w_{u}^{*} & =c_{h_{i}}(u) \\
& \approx \psi_{n}(u) .
\end{aligned}
$$

Thus, by choosing a larger value for $h_{i} \in \Lambda$ in the last application of Lemma 3.3, if necessary, we may assume that

$$
\psi_{n-1}(u) \sim \psi_{n}(u), \quad u \in F_{i}^{0} \backslash F_{i-1}^{0} .
$$

By induction we obtain $\left\{\psi_{n}(e) \mid e \in E^{1}\right\}$ satisfying $\left(1_{n}\right)-\left(6_{n}\right)$ and $(\mathcal{O})$.

Theorem 3.12. If $A$ is a simple separable nuclear purely infinite $C^{*}$-algebra satisfying the Universal Coefficient Theorem, and if A has finitely generated $K$-theory and torsion-free $K_{1}$, then $A$ is semiprojective.

Proof. We rely, of course, on the celebrated theorem of Kirchberg/Phillips [10, [12, stating that simple, separable, nuclear, purely infinite $C^{*}$-algebras with UCT that have isomorphic $K$-theory are strongly Morita equivalent. In addition, we have 
3], which tells us that strongly Morita equivalent separable $C^{*}$-algebras are stably isomorphic.

The case where $\operatorname{rank} K_{0}(A)=\operatorname{rank} K_{1}(A)$ is proved in [1. In the remaining cases, it follows from Theorem 3.1 and Theorem 2.3 that there is a nonunital $C^{*}$ algebra strongly Morita equivalent to $A$ that is semiprojective. Since nonunital simple purely infinite $C^{*}$-algebras are stable (by a theorem of Zhang [20]), it follows that $\mathcal{K} \otimes A$ is semiprojective. Thus we may assume that $A$ is unital.

Let $u_{1}, u_{2}, \ldots$ be isometries in $A$ with pairwise orthogonal final projections. Then $A_{0}=\overline{\operatorname{span}}\left\{u_{i} A u_{j} \mid 1 \leq i, j\right\}$ is a hereditary subalgebra of $A$, and $A_{0} \cong \mathcal{K} \otimes A$. Thus we know that $A_{0}$ is semiprojective.

Now let $B$ be a $C^{*}$-algebra, let $\mathcal{L}$ be a directed family of ideals of $B$ with closure $I$, and suppose that $A=B / I$. Let $\pi: B \rightarrow B / I$ be the quotient map, and set $B_{0}=\pi^{-1}\left(A_{0}\right)$. Since $A_{0}$ is semiprojective there is $J \in \mathcal{L}$ and a $*$-homomorphism $\psi_{0}: A_{0} \rightarrow B_{0} / J$ with $\pi \circ \psi_{0}=\mathrm{id}_{A_{0}}$. We use $\pi_{J}$ to denote the quotient map of $B$ onto $B / J$. Let $v \in B$ with $\pi(v)=u_{1}$. Increasing $J$ if necessary, we may assume that $\pi_{J}\left(v^{*} v\right)=1_{B / J}$ and $\pi\left(v v^{*}\right)=\psi_{0}\left(u_{1} u_{1}^{*}\right)$. Now define $\psi: A \rightarrow B / J$ by

$$
\psi(x)=v^{*} \psi_{0}\left(u_{1} x u_{1}^{*}\right) v .
$$

Then $\psi$ is a $*$-homomorphism, and for $x \in A$,

$$
\begin{aligned}
\pi \circ \psi(x) & =\pi\left(v^{*}\right) \pi \circ \psi_{0}\left(u_{1} x u_{1}^{*}\right) \pi(v) \\
& =u_{1}^{*}\left(u_{1} x u_{1}^{*}\right) u_{1} \\
& =x .
\end{aligned}
$$

Thus $A$ is semiprojective. (See also [11, Proposition 5.48.)

We remark that the final argument in the proof of Theorem 3.12 is a partial converse to a theorem of Blackadar ([2], Theorem 4.1). There he proved (with arguments similar to those in Lemma 3.11) that for properly infinite $C^{*}$-algebras, semiprojectivity passes from a unital algebra to its stabilization.

\section{REFERENCES}

[1] B. Blackadar, Shape theory for $C^{*}$-algebras, Math. Scand. 56 (1985), 249-275. MR813640 (87b:46074)

[2] B. Blackadar, Semiprojectivity in simple $C^{*}$-algebras, Operator algebras and applications, 117, Adv. Stud. Pure Math., 38, Math. Soc. Japan, Tokyo, 2004. MR2059799 (2005g:46101)

[3] L.G. Brown, P. Green and M. Rieffel, Stable isomorphism and strong Morita equivalence of $C^{*}$-algebras, Pacific J. Math. 71 (1977), 349-363. MR0463928 (57:3866)

[4] J. Cuntz, A class of $C^{*}$-algebras and topological Markov chains II: Reducible chains and the Ext-functor for $C^{*}$-algebras, Invent. Math. 63 (1981), 25-40. MR608527 (82f:46073b)

[5] J. Cuntz, $K$-theory for certain $C^{*}$-algebras, Ann. of Math. (2) 113 (1981), 181-197. MR604046 (84c:46058)

[6] J. Cuntz and W. Krieger, A class of $C^{*}$-algebras and topological Markov chains, Invent. Math. 56 (1980), 251-268. MR561974 (82f:46073a)

[7] E.G. Effros and J. Kaminker, Homotopy continuity and shape theory for $C^{*}$-algebras, in Geometric Methods in Operator Algebras, eds. Araki and Effros, Pitman Res. Notes Math. 123, Longman, Harlow, 1986. MR866493 (88a:46082)

[8] R. Exel and M. Laca, Cuntz-Krieger algebras for infinite matrices, J. Reine Angew. Mathematik 512, (1999), 119-172. MR1703078 (2000i:46064)

[9] J. Glimm, On a certain class of operator algebras, Trans. Amer. Math. Soc. 95 (1960), 318-340. MR0112057 (22:2915)

[10] E. Kirchberg, The classification of purely infinite $C^{*}$-algebras using Kasparov's theory, (preprint). 
[11] B. Neubüser, Semiprojektivität und realisierunen von rein unendlichen $C^{*}$-algebren, preprint, Münster, 2000.

[12] N.C. Phillips, A classification theorem for nuclear purely infinite simple $C^{*}$-algebras, Documenta Math. 5 (2000), 49-114. MR1745197(2001d:46086b)

[13] I. Raeburn, Graph Algebras, CBMS Regional Conference Series in Mathematics 103, AMS, Providence, 2005. MR2135030 (2005k:46141)

[14] I. Raeburn and W. Szymanski, Cuntz-Krieger algebras of infinite graphs and matrices, Trans. Amer. Math. Soc. 356 (2004), no. 1, 39-59. MR2020023 (2004i:46087)

[15] M. Rørdam, Classification of Cuntz-Krieger algebras, K-Theory 9 (1995), 31-58. MR.1340839 (96k:46103)

[16] J. Spielberg, A functorial approach to the $C^{*}$-algebras of a graph, Internat. J. Math. 13 (2002), no. 3, 245-277. MR 1911104 (2004e:46084)

[17] J. Spielberg, Non-cyclotomic presentations of modules and prime-order automorphisms of Kirchberg algebras, (preprint, 2005), Mathematics ArXiv OA/0504287. MR2377136

[18] W. Szymanski, On semiprojectivity of $C^{*}$-algebras of directed graphs, Proc. Amer. Math. Soc. 130 (2002), no. 5, 1391-1399. MR1879962(2003a:46083)

[19] W. Szymanski, The range of $K$-invariants for $C^{*}$-algebras of infinite graphs, Indiana Univ. Math. J. 51 (2002), no. 1, 239-249. MR1896162 (2003b:46077)

[20] S. Zhang, Certain $C^{*}$-algebras with real rank zero and their corona and multiplier algebras, Pacific J. Math. 155 (1992), 169-197. MR 1174483 (94i:46093)

Department of Mathematics and Statistics, Arizona State University, Tempe, AriZONA 85287-1804

E-mail address: jack.spielberg@asu.edu 